\title{
Explicit Suboptimal Linear Quadratic Regulation with State and Input Constraints
}

\author{
Tor A. Johansen*,**, Idar Petersen**, and Olav Slupphaug* \\ * Department of Engineering Cybernetics, \\ Norwegian University of Science and Technology, \\ N-7491 Trondheim, Norway. \\ ** SINTEF Electronics and Cybernetics, \\ N-7465 Trondheim, Norway.
}

28 February 2000, SINTEF report STF72-A00303

\begin{abstract}
Optimal feedback solutions to the infinite horizon LQR problem with state and input constraints based on receding horizon real-time quadratic programming are well known. In this paper we develop an explicit solution to the same problem, eliminating the need for realtime optimization. A suboptimal strategy, based on a suboptimal choice of a finite horizon and imposing additional limitations on the allowed switching between active constraint sets on the horizon, is suggested in order to address the computer memory and processing capacity requirements of the explicit solution. It is shown that the resulting feedback controller is piecewise linear, and the piecewise linear structure is explored and exploited for computational analysis of stability and performance of the suboptimal constrained LQR. The piecewise linear structure can also be exploited for efficient real-time implementation of the controller.
\end{abstract}




\section{Contents}

1 Introduction 3

2 Controller Design 5

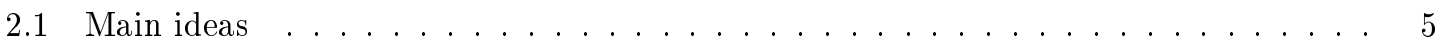

2.2 Decomposition of the HJB equation $\ldots \ldots \ldots \ldots \ldots \ldots$

2.3 Feedback design . . . . . . . . . . . . . . . . . . . . 8

2.4 Recovery from infeasibility . . . . . . . . . . . . . . . . . 10

2.5 Example: Double integrator with input and state constraints . . . . . . . . . . 11

2.6 Avoiding high-gain feedback, chattering and sliding modes . . . . . . . . . . 12

2.7 Setpoint tracking . . . . . . . . . . . . . . . . . . . . 17

3 Real-time Implementation $\quad 17$

4 Piecewise Linear Structure of the Controller $\quad 17$

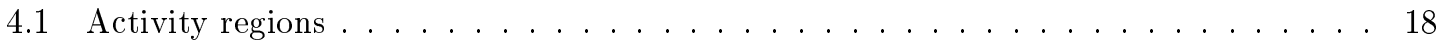

4.2 Outer Approximations to the Activity Regions . . . . . . . . . . . . . . . 20

4.3 Partitioning Algorithm . . . . . . . . . . . . . . . . . . . 23

5 Stability and Performance Analysis $\quad 25$

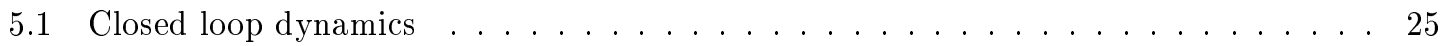

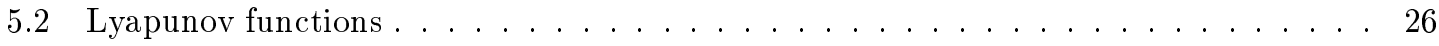

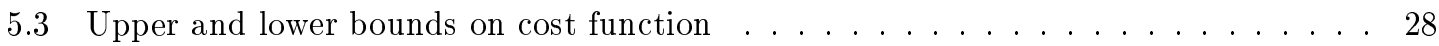

6 Conclusions 28 


\section{Introduction}

Consider the continuous-time linear time-invariant system

$$
\dot{x}=A_{c} x+B_{c} u
$$

and its sampled discrete-time version

$$
x(t+1)=A x(t)+B u(t)
$$

where $x \in R^{n}$, and $u \in R^{r}$. In this paper, the discrete-time version will be used for controller design, while the continuous-time version is used for analysis.

The optimal constrained LQ feedback controller minimizes the infinite horizon quadratic cost

$$
\begin{aligned}
J(u(t), u(t+1), u(t+2), \ldots ; x(t)) & =\sum_{\tau=t}^{\infty} l_{Q R}(x(\tau), u(\tau)) \\
l_{Q R}(x, u) & =x^{T} Q x+u^{T} R u
\end{aligned}
$$

subject to the linear constraints

$$
\begin{aligned}
G x(\tau+1) & \leq g \\
H u(\tau) & \leq h
\end{aligned}
$$

for all $\tau \geq t$, where $R>0, Q \geq 0, G \in R^{q \times n}$, and $H \in R^{p \times r}$. It is assumed that $g, h>0$ to ensure that the origin is an interior point in the admissible region. The optimal cost function is defined as

$$
V(x(t))=\min _{u(t), u(t+1), \ldots} J(u(t), u(t+1), u(t+2), \ldots ; x(t))
$$

where the minimization is subject to the dynamics of the system (2), and the constraints (5)-(6) are imposed at every time instant $\tau \in\{t, t+1, t+2, \ldots\}$ on the trajectory. The cost of moving from the state $x(t)$ to the origin in an optimal manner is given by $V(x(t))$. The Hamilton-Jacobi-Bellman (HJB) equation characterizes the optimal cost function and optimal control action for the problem (Bellman 1957):

$$
0=\min _{\substack{u(\tau) \in R^{r}, G x(\tau+1) \leq g, H u(\tau) \leq h \\ \tau \in\{t, t+1, t+2, \ldots, t+N-1\}}}\left(V(x(t+N))-V(x(t))+\sum_{\tau=t}^{t+N-1} l_{Q R}(x(\tau), u(\tau))\right)
$$

where $N \geq 1$ is some horizon, and $V(0)=0$. Under the assumptions of feasibility, non-explicit optimal solutions to the HJB (8) are known for the case when $N$ is so large that there are no active or violated constraints beyond this horizon and the unconstrained LQ solution is optimal beyond the horizon (Sznaier and Damborg 1987, Chmielewski and Manousiouthakis 1996, Scokaert and Rawlings 1998). These solutions are based on real-time quadratic programming, where a finitedimensional optimization problem is achieved since $V(x(t+N))=x^{T}(t+N) P x(t+N)$, where $P$ is the solution to the Ricatti equation associated with the unconstrained LQR. An algorithm to compute the sufficiently large $N$ can be found in (Chmielewski and Manousiouthakis 1996). This is an optimal approach, in contrast to common suboptimal (approximate) approaches used in model predictive control with a finite horizon cost function approximation or a finite input move horizon, e.g. (Keerthi and Gilbert 1988, Rawlings and Muske 1993). In all the above mentioned approaches the controller is implicit and computation of the control input $u(t)$ relies on real-time quadratic programming at each sample. This imposes severe limitations on the achievable sample rate that may discourage the application of this highly successful approach in many cases.

Recently, in some unpublished work, Bemporad et al. (1999) derived an optimal explicit solution to the constrained LQR problem using offline multi-parametric quadratic programming. The constrained LQR problem is viewed as a quadratic program parameterized by the state $x$, and the multi-parametric quadratic programming approach essentially finds an explicit solution for all $x$ within an arbitrary subset of the state space. Again, the optimal solution requires that $N$ is sufficiently large. The resulting optimal controller was proved to be a piecewise linear function defined on a polyhedral partitioning of the state-space. 
In this paper we also seek an explicit solution (in the sense that no real-time quadratic program needs to be solved) to this problem in order to reduce the demand for real-time computations. However, in order to address the constraints imposed by the real-time application on both computer memory and processing capacity, a (possibly) suboptimal strategy is developed. Hence, one has a mechanism to trade performance for computational advantages. Moreover, the problem formulation is extended from the basic formulation (2)-(6) by introducing two additional specifications:

1. It is useful to allow the constraint limits $g, h$ to be modified on-line, for example to meet variations in operating conditions, setpoint changes or controller reconfiguration due to faults. Hence, we allow $g \in \mathcal{G}$ and $h \in \mathcal{H}$, where $\mathcal{G}$ and $\mathcal{H}$ are polyhedral sets.

2. Due to input constraints, unmodelled dynamics, disturbances, noise and suboptimal control, the state may move out of the admissible state-space region where $G x \leq g$. Thus, we introduce one additional specification that will be formulated more precisely in later sections. If no feasible control input that prevents the state from moving into the non-admissible region on the prediction horizon can be computed, the LQ objective is subsumed by the more important objective of minimizing the constraint violations according to some criterion that is defined using weighting and prioritization among the violated state constraints.

Both these additional specification are similar to what is being applied in model predictive control, e.g. (Rawlings and Muske 1993). The present approach can be seen as an extension of (Bemporad et al. 1999) (although the main parts was developed independently) with the following main differences:

- Here we consider a suboptimal strategy where an approximation to the optimal cost function is utilized and we impose restrictions on the allowed switching between the active constraint sets during the prediction horizon. The motivation for this is that in some applications, implementation of the optimal explicit solution tends to require large amounts of real-time computer memory or processing capacity. Thus, it is of interest to consider suboptimal approaches of reduced complexity. As a special case, the presented approach degenerates to the optimal explicit LQR of Bemporad et al. (1999).

- Due to the suboptimality of the controller, its performance is not known a priori, so we also provide computational analysis tools which can be used to compute upper and lower bounds on suboptimal performance as well as assess stability based on (Johansson and Rantzer 1998, Rantzer and Johansson 1997). Note that also the approach in (Bemporad et al. 1999) may be suboptimal if the prediction horizon is not sufficiently large to guarantee that no constraints are violated beyond it.

- Both strategies leads to a piecewise linear (PWL) controller. While the exact approach leads to a continuous PWL function on a polyhedral partitioning, the suboptimal approach will not do so in all cases.

- The present approach explicitly addresses the possibility of infeasibility in the design and analysis.

- Our formulation allows the constraint limits to be modified by the operator or a supervisory controller on-line, for example in order to adapt the constraints to a new setpoint, without requiring recomputation of the controller parameters or drastically increasing the need for real-time computer memory or processing capacity.

- The present design approach includes practical modifications to avoid high gain feedback (which may result in sliding mode like behaviour and chattering control) at the boundary of the state constraints.

- As illustrated by the two points above, we believe the suboptimal formulation in general allows for more flexibility in implementing and analysing modifications to the original constrained LQR problem or solution.

- The solution strategies are different; the present approach is not based on multi-parametric quadratic programming. There appears to exist no multi-parametric quadratic program that is equivalent to the suboptimal strategy proposed here. 
In (Chisci 1999), the structure of the finite-dimensional real-time quadratic program is utilized to developed a fast QP algorithm based on active sets for constrained LQR. In an alternative approach, (Sznaier and Damborg 1990), a finite discrete search problem is achieved by quantization of the set of admissible inputs. The computational complexity of their search problem is typically much larger, and a different type of approximation is introduced due to the quantization of the inputs. The approach of (Wredenhagen and Belanger 1994) defines a nested set of elliptic regions of the state space, each containing the origin, where different LQ optimal feedback laws are designed with different $Q$ matrices. The idea is to reduce the gain of the feedback in order to avoid saturation when far away from the origin. However, only input constraints are considered.

\section{Controller Design}

\subsection{Main ideas}

Let $D^{T}=\left(d_{1}, d_{2}, \ldots, d_{m}\right)$ and $e^{T}=\left(e_{1}, e_{2}, \ldots, e_{n}\right)$. A single inequality constraint $d_{i}^{T} z \geq e_{i}$ is said to be an active constraint if $d_{i}^{T} z=e_{i}$, where $d_{i}$ is a vector, $e_{i}$ is a scalar and the vector $z$ is the design variable. An active constraint set associated with some set of inequality constraints $D z \leq e$ is the set of indices to those constraints that are active. Note that the active constraint set may be empty, meaning that no constraints are active.

At each sample one may impose a number of equality constraints on the states and inputs that at most is equal to the number of inputs $r$. This selection of constraints is the active constraint set associated with that sample. A sequence of active constraint sets imposed at each sample on the horizon finite $N$ is called an active constraint set sequence. A naive solution strategy to the optimal explicit LQR problem is now simply to evaluate all feasible active constraint set sequences on a sufficiently large horizon $N$ in order to see which one is optimal. This naive solution strategy to the optimal explicit LQR may have offline computational disadvantages compared to the multi-parametric quadratic programming approach of (Bemporad et al. 1999) since the number of candidate active constraint set sequences increases very rapidly with the horizon $N$, the number of inputs $r$ and states $n$. However, it has the advantage that it can be easily modified to determine suboptimal explicit LQR solutions with drastically reduced offline and real-time computational demands. Here we suggest to use a smaller horizon $N$ than required by the optimal solution and in addition to reduce the flexibility in the active constraint set sequence by allowing only a smaller number of changes in the active constraint set on the horizon $N$. Furthermore, one may require that the active constraint set changes are made at predetermined (fixed) samples. For example, one may subdivide the horizon $N=10$ into $S=3$ subintervals, and only allow the active constraint set to change at the beginning of each subinterval. Note that this subinterval strategy is fundamentally different from the model predictive control approach. In model predictive control it is common to impose restrictions on the number of input changes that are allowed on the horizon, again at fixed samples. This will make the control input constant within each subinterval, while the strategy suggested in the present paper will make the affine state-feedback control law associated with each active constraint set constant within each subinterval. This is expected to give a more flexible and less suboptimal parameterization.

Suppose the set of indices $\alpha$ is associated with the active input constraints in (6) at some sample, and the set of indices $\beta$ is associated with the active state constraints (5) at the same sample. Then $(\alpha, \beta)$ is an active constraint set. Next, suppose we define allowed switching times as follows

$$
0=N_{1}<N_{2}<\cdots<N_{S}<N
$$

For example, if $S=3, N_{1}=0, N_{2}=3$ and $N_{3}=7$ there will be 3 subintervals $\{t, t+1, t+2\}$, $\{t+3, t+4, t+5, t+6\}$, and $\{t+7, t+8, t+9\}$ with associated fixed active constraint sets $\left(\alpha_{1}, \beta_{1}\right),\left(\alpha_{2}, \beta_{2}\right),\left(\alpha_{3}, \beta_{3}\right)$, respectively. In general, these active constraint sets lead to an active constraint set sequence $\left(\left(\alpha_{1}, \beta_{1}\right),\left(\alpha_{2}, \beta_{2}\right), \ldots,\left(\alpha_{N_{S}}, \beta_{N_{S}}\right)\right)$ that together with $\left(N_{1}, N_{2}, \ldots, N_{S}\right)$ and $N$ define the active constraint set imposed at each sample on the horizon. This means that the constraints indexed by each active constraint set are imposed on the associated interval, leading to 
the following set of equality constraints:

$$
\left.\begin{array}{c}
G_{\beta_{1}} x(t+1)=g_{\beta_{1}} \\
G_{\beta_{1}} x(t+2)=g_{\beta_{1}} \\
\vdots \\
G_{\beta_{1}} x\left(t+N_{2}-1\right)=g_{\beta_{1}} \\
G_{\beta_{2}} x\left(t+N_{2}\right)=g_{\beta_{2}} \\
G_{\beta_{2}} x\left(t+N_{2}+1\right)=g_{\beta_{2}} \\
\vdots \\
G_{\beta_{2}} x\left(t+N_{3}-1\right)=g_{\beta_{2}} \\
G_{\beta_{3}} x\left(t+N_{3}\right)=g_{\beta_{3}} \\
\vdots \\
G_{\beta_{N_{S}}} x(t+N)=g_{\beta_{N_{S}}}
\end{array}\right\}
$$

and

$$
\left.\begin{array}{c}
H_{\alpha_{1}} u(t)=h_{\alpha_{1}} \\
H_{\alpha_{1}} u(t+1)=h_{\alpha_{1}} \\
\vdots \\
H_{\alpha_{1}} u\left(t+N_{2}-2\right)=h_{\alpha_{1}} \\
H_{\alpha_{2}} u\left(t+N_{2}-1\right)=h_{\alpha_{2}} \\
H_{\alpha_{2}} u\left(t+N_{2}\right)=h_{\alpha_{2}} \\
\vdots \\
H_{\alpha_{2}} u\left(t+N_{3}-2\right)=h_{\alpha_{2}} \\
H_{\alpha_{3}} u\left(t+N_{3}-1\right)=h_{\alpha_{3}} \\
\vdots \\
H_{\alpha_{N_{S}}} u(t+N-1)=h_{\alpha_{N_{S}}}
\end{array}\right\}
$$

Note that the input constraints are imposed at the current and future samples $\{t, t+1, \ldots, t+N-1\}$ while the state constraints are imposed at future samples $\{t+1, t+2, \ldots, t+N\}$. Introducing the matrix

$$
C_{\tau}=\left(A^{\tau-1} B\left|A^{\tau-2} B\right| \cdots \mid B\right)
$$

and the $r N \times r N$-matrix $\tilde{E}_{\tau}$ defined by

$$
\tilde{E}_{\tau}=\left(\begin{array}{cc}
0 & 0 \\
I_{r \tau \times r \tau} & 0
\end{array}\right)
$$

we establish the well known formula

$$
x(t+\tau)=A^{\tau} x(t)+C_{N} \tilde{E}_{\tau} \tilde{u}(t)
$$


where $\tilde{u}(t)=\left(u^{T}(t), u^{T}(t+1), \vdots, u^{T}(t+N-1)\right)^{T}$. Combining (14) and (10) we get

$$
\left.\begin{array}{c}
G_{\beta_{1}}\left(A x(t)+C_{N} \tilde{E}_{1} \tilde{u}(t)\right)=g_{\beta_{1}} \\
G_{\beta_{1}}\left(A^{2} x(t)+C_{N} \tilde{E}_{2} \tilde{u}(t)\right)=g_{\beta_{1}} \\
\vdots \\
G_{\beta_{1}}\left(A^{N_{2}-1} x(t)+C_{N} \tilde{E}_{N_{2}-1} \tilde{u}(t)\right)=g_{\beta_{1}} \\
G_{\beta_{2}}\left(A^{N_{2}} x(t)+C_{N} \tilde{E}_{N_{2}} \tilde{u}(t)\right)=g_{\beta_{2}} \\
G_{\beta_{2}}\left(A^{N_{2}+1} x(t)+C_{N} \tilde{E}_{N_{2}+1} \tilde{u}(t)\right)=g_{\beta_{2}} \\
\vdots \\
G_{\beta_{2}}\left(A^{N_{3}-1} x(t)+C_{N} \tilde{E}_{N_{3}-1} \tilde{u}(t)\right)=g_{\beta_{2}} \\
G_{\beta_{3}}\left(A^{N_{3}} x(t)+C_{N} \tilde{E}_{N_{3}} \tilde{u}(t)\right)=g_{\beta_{3}} \\
\vdots \\
G_{\beta_{N_{S}}}\left(A^{N} x(t)+C_{N} \tilde{E}_{N} \tilde{u}(t)\right)=g_{\beta_{N_{S}}}
\end{array}\right\}
$$

Removing from (11) and (15) equations that are a priori known to be infeasible and duplicated equations, (11) and (15) may be stacked into the following set of equations

$$
L_{k} \tilde{u}(t)=M_{k} x(t)+M_{k}^{g} g+M_{k}^{h} h
$$

where $k$ is an index in the index set $\mathcal{C}=\left\{0,1,2, \ldots, N_{K}-1\right\}$ enumerating the finite set of all active constraint set sequences generated by the constraints (6), (5) and the division into subintervals (9). Let $k_{0} \in \mathcal{C}$ be the index to the active constraint set sequence with no active constraints.

\subsection{Decomposition of the HJB equation}

The minimization problem on the RHS of (8) with the stated constraints is a convex problem whose solution is characterized by the Karush-Kuhn-Tucker conditions. However, since these conditions involve inequalities, the Karush-Kuhn-Tucker conditions provide an implicit solution that does not lead to an explicit state-feedback implementation of the controller. This motivates a simple reformulation of the minimization in (8) into two nested parts where one part only involves equality contraints and the other part is a discrete optimization problem over all allowed active constraint set sequences. The part that involves equality constraints can then be solved explicitly offline using Lagrange multipliers, while the discrete optimization problem can in many cases also be solved offline or at least reduced to a simpler problem and then solved in real-time in a efficient manner. Next, define the $r \times r N$ matrix

$$
E_{\tau}=\left(0_{r \times r}, \ldots, 0_{r \times r}, I_{r \times r}, 0_{r \times r}, \ldots 0_{r \times r}\right)
$$

where the $I_{r \times r}$ is at the $\tau$-th $r \times r$ block. The following result is then evident from (Chmielewski and Manousiouthakis 1996):

Theorem 1 (Nested HJB equation) Assume the minimum in the HJB equation (8) exists. With $N$ sufficiently large and no restrictions on the active constraint set sequence's allowed switching times on the horizon $(S=N)$, the HJB equation (8) is equivalent to

$0=\min _{k \in \mathcal{C}}\left(\min _{\substack{\tilde{u}(t) \in R^{r N} \\ L_{k} \tilde{u}=M_{k} x(t)+M_{k}^{g} g+M_{k}^{h} h}}\left(V(x(t+N))-V(x(t))+\sum_{\tau=0}^{N-1} l_{Q R}\left(x(t+\tau), E_{\tau+1} \tilde{u}(t)\right)\right)\right)$

where the outer minimization is subject to the constraints

$$
\begin{aligned}
H E_{\tau} \tilde{u}_{k}^{*}(x(t), g, h) & \leq h \\
G\left(A^{\tau} x(t)+C_{N} \tilde{E}_{\tau} \tilde{u}_{k}^{*}(x(t), g, h)\right) & \leq g
\end{aligned}
$$

for all $1 \leq \tau \leq N$ and $\tilde{u}_{k}^{*}(x(t), g, h)$ is the $\tilde{u}(t)$ solving the innermost optimization problem in (18). 
The inner optimization in (18) assumes a fixed active constraint set sequence and is therefore subject to equality constraints rather than inequality constraints as in (8). Hence, for all fixed active constraint set sequences, affine static state feedback controllers can be explicitly computed offline and stored in computer memory for real-time use, as we shall see. Determining the optimal cost function $V$ is in general an extremely difficult problem, so similar to (Rantzer and Johansson 1997) we utilize a lower bound $\underline{V}$ as a suboptimal approximation in the control design in Section 2.3, and analyse the loss of performance resulting from this approximation as well as suboptimality due to restrictions on the allowed active constraint set switching times later in Section 5. Notice that this lower bound is exact when no constraints are active on any part of the trajectory.

Lemma 1 A lower bound on the optimal cost function is given by $\underline{V}(x)=x^{T} \underline{P} x \leq V(x)$ where the matrix $\underline{P}=\underline{P}^{T}$ is the positive definite solution of the algebraic Ricatti equation corresponding to the unconstrained $L Q R$ problem:

$$
A^{T} \underline{P} A-\underline{P}-A^{T} \underline{P} B\left(B^{T} \underline{P} B+R\right)^{-1} B^{T} \underline{P} A+Q=0
$$

Proof. The result follows immediately from the observation that constraining the input will never decrease the value of the optimal cost function, see also (Sznaier and Damborg 1990) where a similar result is provided.

\subsection{Feedback design}

Consider the innermost optimization on the RHS of (18) with the lower bound $\underline{V}$ substituted for $V$. This problem can be stated as follows: For a given active constraint set sequence (with index $k \in \mathcal{C})$ solve the problem

$$
\tilde{u}_{k}^{*}(x(t), g, h)=\arg \min _{\substack{\tilde{u}(t) \in R^{r N} \\ L_{k} \tilde{u}(t)=M_{k} x(t)+M_{k}^{g} g+M_{k}^{h} h}} \underline{I}(\tilde{u}(t), x(t))
$$

where

$$
\underline{I}(\tilde{u}(t), x(t))=\underline{V}(x(t+N))-\underline{V}(x(t))+\sum_{\tau=0}^{N-1} l_{Q R}\left(x(t+\tau), E_{\tau+1} \tilde{u}(t)\right)
$$

The outer finite discrete optimization problem of (18) can be restated as

$$
\begin{aligned}
k^{*}(x, g, h) & =\arg \min _{k \in \mathcal{C}} \varphi_{k}(x, g, h) \\
\varphi_{k}(x, g, h) & =\underline{I}\left(\tilde{u}_{k}^{*}(x, g, h), x\right)
\end{aligned}
$$

where the minimization is subject to

$$
\begin{aligned}
H E_{\tau} \tilde{u}_{k}^{*}(x, g, h) & \leq h \\
G\left(A^{\tau} x+C_{N} \tilde{E}_{\tau} \tilde{u}_{k}^{*}(x, g, h)\right) & \leq g
\end{aligned}
$$

for all $1 \leq \tau \leq N$. Note that the minimum $k^{*}(x, g, h)$ need not be unique. In this case $k^{*}(x, g, h)$ is selected according to some preordering of the candidate minima. Eq. (26) states that the control input given by the feedback must be feasible with respect to the input constraints. Eq. (27) states that the state must stay in the admissible region.

The optimization problem (24)-(27) is feasible if and only if $(x, g, h) \in Z^{F}$ :

$$
\begin{aligned}
Z^{F}= & \bigcup_{k \in \mathcal{C}} Z_{k}^{F} \\
Z_{k}^{F}= & \left\{(x, g, h) \in R^{n} \times \mathcal{G} \times \mathcal{H} \mid\right. \\
& \left.H E_{\tau} \tilde{u}_{k}^{*}(x, g, h) \leq h, G\left(A^{\tau} x+C_{N} \tilde{E}_{\tau}^{T} \tilde{u}_{k}^{*}(x, g, h)\right) \leq g, \text { for } 1 \leq \tau \leq N\right\}
\end{aligned}
$$


When $(g, h)$ are fixed (i.e. $\mathcal{G}$ and $\mathcal{H}$ degenerate to single points), we define $X^{F}(g, h)$ as the projection of $Z^{F}$ into $R^{n}$, and likewise for $X_{k}^{F}(g, h)$. Outside the feasible region $Z^{F}$ an alternative strategy should be applied. This is the topic of section 2.4. For $(x, g, h) \in Z^{F}$, the suboptimal constrained LQR is now given by

$$
u^{*}(x, g, h)=E_{1} \tilde{u}_{k^{*}(x, g, h)}^{*}(x, g, h)
$$

Next, we reformulate the expression (23) for $\underline{I}$. We use

$$
\begin{aligned}
\underline{V}(x(t+N))= & \left(x^{T}(t)\left(A^{T}\right)^{N}+\tilde{u}^{T}(t) C_{N}^{T}\right) \underline{P}\left(A^{N} x(t)+C_{N} \tilde{u}(t)\right) \\
l_{Q R}\left(x(t+\tau), E_{\tau+1} \tilde{u}(t)\right)= & \left(x^{T}(t)\left(A^{\tau}\right)^{T}+\tilde{u}^{T}(t) \tilde{E}_{\tau}^{T} C_{N}^{T}\right) Q\left(A^{\tau} x(t)+C_{N} \tilde{E}_{\tau} \tilde{u}(t)\right) \\
& +\tilde{u}^{T}(t) E_{\tau+1}^{T} R E_{\tau+1} \tilde{u}(t)
\end{aligned}
$$

for $0 \leq \tau \leq N-1$, and we get (note that $\tilde{E}_{0}=0$ )

$$
\begin{aligned}
\underline{I}(\tilde{u}, x)=x^{T} & \left(Q+A^{T} Q A+\left(A^{2}\right)^{T} Q A^{2}+\ldots+\left(A^{N-1}\right)^{T} Q A^{N-1}+\left(A^{N}\right)^{T} \underline{P} A^{N}-\underline{P}\right) x \\
+ & 2 x^{T}\left(A^{T} Q C_{N} \tilde{E}_{1}+\ldots+\left(A^{N-1}\right)^{T} Q C_{N} \tilde{E}_{N-1}+\left(A^{N}\right)^{T} \underline{P} C_{N}\right) \tilde{u} \\
+ & \tilde{u}^{T}\left(\tilde{R}+\tilde{E}_{1}^{T} C_{N}^{T} Q C_{N} \tilde{E}_{1}+\ldots+\tilde{E}_{N-1}^{T} C_{N}^{T} Q C_{N} \tilde{E}_{N-1}+C_{N}^{T} \underline{P} C_{N}\right) \tilde{u}
\end{aligned}
$$

where the $r N \times r N$-matrix $\tilde{R}$ is defined by

$$
\tilde{R}=\left(\begin{array}{cccc}
R & 0 & \cdots & 0 \\
0 & R & \cdots & 0 \\
\vdots & \vdots & & \vdots \\
0 & 0 & \cdots & R
\end{array}\right)
$$

Defining

$$
\begin{aligned}
& S_{1}=Q+A^{T} Q A+\left(A^{2}\right)^{T} Q A^{2}+\ldots+\left(A^{N-1}\right)^{T} Q A^{N-1}+\left(A^{N}\right)^{T} \underline{P} A^{N}-\underline{P} \\
& S_{2}=A^{T} Q C_{N} \tilde{E}_{1}+\ldots+\left(A^{N-1}\right)^{T} Q C_{N} \tilde{E}_{N-1}+\left(A^{N}\right)^{T} \underline{P} C_{N} \\
& S_{3}=\tilde{R}+\tilde{E}_{1}^{T} C_{N}^{T} Q C_{N} \tilde{E}_{1}+\ldots+\tilde{E}_{N-1}^{T} C_{N}^{T} Q C_{N} \tilde{E}_{N-1}+C_{N}^{T} \underline{P} C_{N}
\end{aligned}
$$

we get the compact expression

$$
\underline{I}(\tilde{u}, x)=x^{T} S_{1} x+2 x^{T} S_{2} \tilde{u}+\tilde{u}^{T} S_{3} \tilde{u}
$$

Theorem 2 (Piecewise linear state feedback design) Consider a fixed active constraint set sequence with index $k \in \mathcal{C}$. The solution to the constrained quadratic optimization problem (22) is given by the affine state feedback

$$
\tilde{u}_{k}^{*}(x, g, h)= \begin{cases}K_{k, 2} x, & \text { if } k=k_{0} \\ K_{k, 1}^{g} g+K_{k, 1}^{h} h+K_{k, 2} x, & \text { if } k \neq k_{0}\end{cases}
$$

where $K_{k, 2}=-S_{3}^{-1} S_{2}^{T}$ for $k=k_{0}$ and

$$
\begin{aligned}
K_{k, 1}^{g} & =S_{3}^{-1} L_{k}^{T}\left(L_{k} S_{3}^{-1} L_{k}^{T}\right)^{-1} M_{k}^{g} \\
K_{k, 1}^{h} & =S_{3}^{-1} L_{k}^{T}\left(L_{k} S_{3}^{-1} L_{k}^{T}\right)^{-1} M_{k}^{h} \\
K_{k, 2} & =-S_{3}^{-1}\left(\left(I-L_{k}^{T}\left(L_{k} S_{3}^{-1} L_{k}^{T}\right)^{-1} L_{k} S_{3}^{-1}\right) S_{2}^{T}-L_{k}^{T}\left(L_{k} S_{3}^{-1} L_{k}^{T}\right)^{-1} M_{k}\right)
\end{aligned}
$$

for $k \neq k_{0}$ and $(x, g, h) \in Z^{F}$.

Proof. Note that (22) is convex since $\tilde{R}>0$. The special case when $k=k_{0}$ follows directly from unconstrained minimization. Next, consider the general case $k \neq k_{0}$. Introducing the Lagrange multiplier $\lambda_{k}(x, g, h)$ and minimizing the right hand side expression in (22) leads to the following expression for the optimal control $\tilde{u}_{k}^{*}(x, g, h)$ :

$$
2 S_{2}^{T} x+2 S_{3} \tilde{u}_{k}^{*}(x, g, h)+L_{k}^{T} \lambda_{k}(x, g, h)=0
$$


which can be rewritten

$$
\tilde{u}_{k}^{*}(x, g, h)=-S_{3}^{-1}\left(S_{2}^{T} x+\frac{1}{2} L_{k}^{T} \lambda_{k}(x, g, h)\right)
$$

where the inverse of $S_{3}$ exists since $\tilde{R}$ is positive definite and the 2 nd term in the expression for $S_{3}$ is positive semi-definite. Substituting this expression into the constraint $L_{k} \tilde{u}=M_{k} x+M_{k}^{g} g+M_{k}^{h} h$ gives

$$
-L_{k} S_{3}^{-1}\left(S_{2}^{T} x+\frac{1}{2} L_{k}^{T} \lambda_{k}(x, g, h)\right)=M_{k} x+M_{k}^{g} g+M_{k}^{h} h
$$

This equation can be solved for $\lambda_{k}(x, g, h)$ :

$$
\lambda_{k}(x, g, h)=-2\left(L_{k} S_{3}^{-1} L_{k}^{T}\right)^{-1}\left(\left(M_{k}+L_{k} S_{3}^{-1} S_{2}^{T}\right) x+M_{k}^{g} g+M_{k}^{h} h\right)
$$

where the inverse exists due to the linear independence of the rows of $L_{k}$. By substituting (46) back into (44), the stated affine state feedback is derived

$$
\begin{aligned}
\tilde{u}_{k}^{*}(x, g, h)=- & S_{3}^{-1}\left(S_{2}^{T}-L_{k}^{T}\left(L_{k} S_{3}^{-1} L_{k}^{T}\right)^{-1}\left(M_{k}+L_{k} S_{3}^{-1} S_{2}^{T}\right)\right) x \\
& +S_{3}^{-1} L_{k}^{T}\left(L_{k} S_{3}^{-1} L_{k}^{T}\right)^{-1}\left(M_{k}^{g} g+M_{k}^{h} h\right)
\end{aligned}
$$

Observe that in the case of no active constraints, then (39) takes the form of the well known unconstrained LQR solution, namely $u=-\left(B^{T}(Q+\underline{P}) B+R\right)^{-1} B^{T}(Q+\underline{P}) A x$.

The affine state feedback (39) is parameterized such that individual constraints can be deactivated and the constraint limits may be changed on-line without changing the parameters of the controller. The reason for this is that the constraint limits appear only in $g, h$, and that the precomputed feedback matrices $K_{k, 1}^{g}, K_{k, 1}^{h}$ and $K_{k, 2}$ do not depend on these limits.

\subsection{Recovery from infeasibility}

The optimization problem (24)-(27) will not have solutions that are feasible when $(x, g, h) \notin Z^{F}$. Then one may relax the problem by allowing minimum violation of some of the constraints according to some prioritizations. Constraints that may be relaxed are called "soft" constraints (with indices in the constraint sets $\alpha_{\text {soft }}$ and $\beta_{\text {soft }}$ ), as opposed to "hard" constraints (with indices in the constraint sets $\alpha_{\text {hard }}$ and $\beta_{\text {hard }}$ ), which can not be relaxed under any circumstances. Hence, for $(x, g, h) \notin Z^{F}$, one might minimize the criterion

$$
\begin{aligned}
\nu_{k}(x, g, h)= & \sum_{\tau=1}^{N} \omega_{1, \beta_{s o f t}}^{T} \max \left(0, G_{\beta_{s o f t}}\left(A^{\tau} x+C_{N} \tilde{E}_{\tau} \tilde{u}_{k}^{*}(x, g, h)\right)-g_{\beta_{s o f t}}\right) \\
& +\sum_{\tau=1}^{N} \omega_{2, \alpha_{s o f t}}^{T} \max \left(0, H_{\alpha_{\text {soft }}} E_{\tau} \tilde{u}_{k}^{*}(x, g, h)-h_{\alpha_{\text {sof }}}\right)
\end{aligned}
$$

subject to "hard" constraints for $1 \leq \tau \leq N$

$$
\begin{aligned}
G_{\beta_{\text {hard }}}\left(A^{\tau} x+C_{N} \tilde{E}_{\tau} \tilde{u}_{k}^{*}(x, g, h)\right) & \leq g_{\beta_{\text {hard }}} \\
H_{\alpha_{\text {hard }}} E_{\tau} \tilde{u}_{k}^{*}(x, g, h) & \leq h_{\alpha_{\text {hard }}}
\end{aligned}
$$

with respect to $k \in \mathcal{C}$. The constant positive vectors $\omega_{1, \alpha_{\text {soft }}}$ and $\omega_{2, \beta_{\text {soft }}}$ are weights that capture some prioritization among the soft constraints. The optimization problem (48)-(50) is feasible when $(x, g, h) \in Z^{R}$, where

$$
\begin{aligned}
Z^{R} & =\bigcup_{k \in \mathcal{C}} Z_{k}^{R} \\
Z_{k}^{R} & =\left\{(x, g, h) \in R^{n} \times \mathcal{G} \times \mathcal{H}-Z^{F} \mid \text { such that }(49)-(50) \text { holds }\right\}
\end{aligned}
$$


When $(g, h)$ are fixed we define $X^{R}(g, h)$ as the projection of $Z^{R}$ into $R^{n}$, and likewise for $X_{k}^{R}(g, h)$. If $(x, g, h) \notin Z^{F} \cup Z^{R}$, i.e. no active constraint set sequence in $\mathcal{C}$ gives a control input that is feasible with respect to the hard (non-relaxable) constraints on the horizon, the controller fails. Let the solution to (48)-(50) be denoted $k^{*}(x, g, h)$ and the associated control input $\tilde{u}_{k^{*}(x, g, h)}^{*}(x, g, h)$. Furthermore, let $Z=Z^{F} \cup Z^{R}$ and define for $(x, g, h) \in Z$ the control input of the suboptimal constrained LQR with infeasibility handler:

$$
u^{*}(x, g, h)=E_{1} \tilde{u}_{k^{*}(x, g, h)}^{*}(x, g, h)
$$

\subsection{Example: Double integrator with input and state constraints}

Consider the continuous-time double integrator

$$
A_{c}=\left(\begin{array}{cc}
0 & 1 \\
0 & 0
\end{array}\right), \quad B_{c}=\left(\begin{array}{c}
0 \\
1
\end{array}\right)
$$

with the discretized model

$$
A=\left(\begin{array}{cc}
1 & T_{s} \\
0 & 1
\end{array}\right), \quad B=\left(\begin{array}{c}
T_{s}^{2} \\
T_{s}
\end{array}\right)
$$

using a sampling-interval $T_{s}=0.05$. The control objective is defined by the cost function

$$
l_{Q R}(x, u)=x_{1}^{2}+u^{2}=x^{T}\left(\begin{array}{ll}
1 & 0 \\
0 & 0
\end{array}\right) x+u^{2}
$$

and the constraints

$$
\begin{aligned}
-0.5 & \leq x_{2} \leq 0.5 \\
-1 & \leq u \leq 1
\end{aligned}
$$

If $x_{1}$ is interpreted as position, $x_{2}$ as speed and $u$ as force, the objective is to control the position under constraints on the speed and force. The state constraint will be of the form (5) by defining

$$
G=\left(\begin{array}{cc}
0 & 1 \\
0 & -1
\end{array}\right), \quad g=\left(\begin{array}{c}
0.5 \\
0.5
\end{array}\right)
$$

and the input constraints of the form

$$
H=\left(\begin{array}{c}
1 \\
-1
\end{array}\right), \quad h=\left(\begin{array}{l}
1 \\
1
\end{array}\right)
$$

In this example the constraint limits $g$ and $h$ are assumed to be fixed. Figure 1 shows a simulation when the initial state is $x(0)=(-2,0)^{T}$. Observe that initially the input constraint $u=1$ is active. After $t \approx 0.5$, the state constraint $x_{2} \leq 0.5$ is active, until $t \approx 2.85$ when the controller switches strategy once more, since it appears to be no longer optimal to stay on the constraint $x_{2}=0.5$. After this point the unconstrained LQ controller is used and the state is controlled to the origin. The switching strategy chosen by the controller is intuitive: In order to reduce the position error the speed is first increased at a maxmimum rate (given by the input constraint). When the maximum speed allowed is reached, this speed is kept until the position error becomes so small that the speed must be reduced to stabilize the position at the setpoint. In this example we have choosen the smallest possible horizon, namely $N=S=1$ since this is advantageous for computational reasons. However, at least for this simulation, this extreme choice does not appear to be significantly suboptimal.

Now, the region of feasibility is

$$
X^{F}=\left\{x \in R^{2}|| x_{2} \mid \leq 0.55\right\}
$$

since the input constraints restricts $x_{2}$ to be changed by at most 0.05 units within one sample. Hence, the admissible region $\left|x_{2}\right| \leq 0.5$ can be reached in one sample from $X^{F}$. In order to 


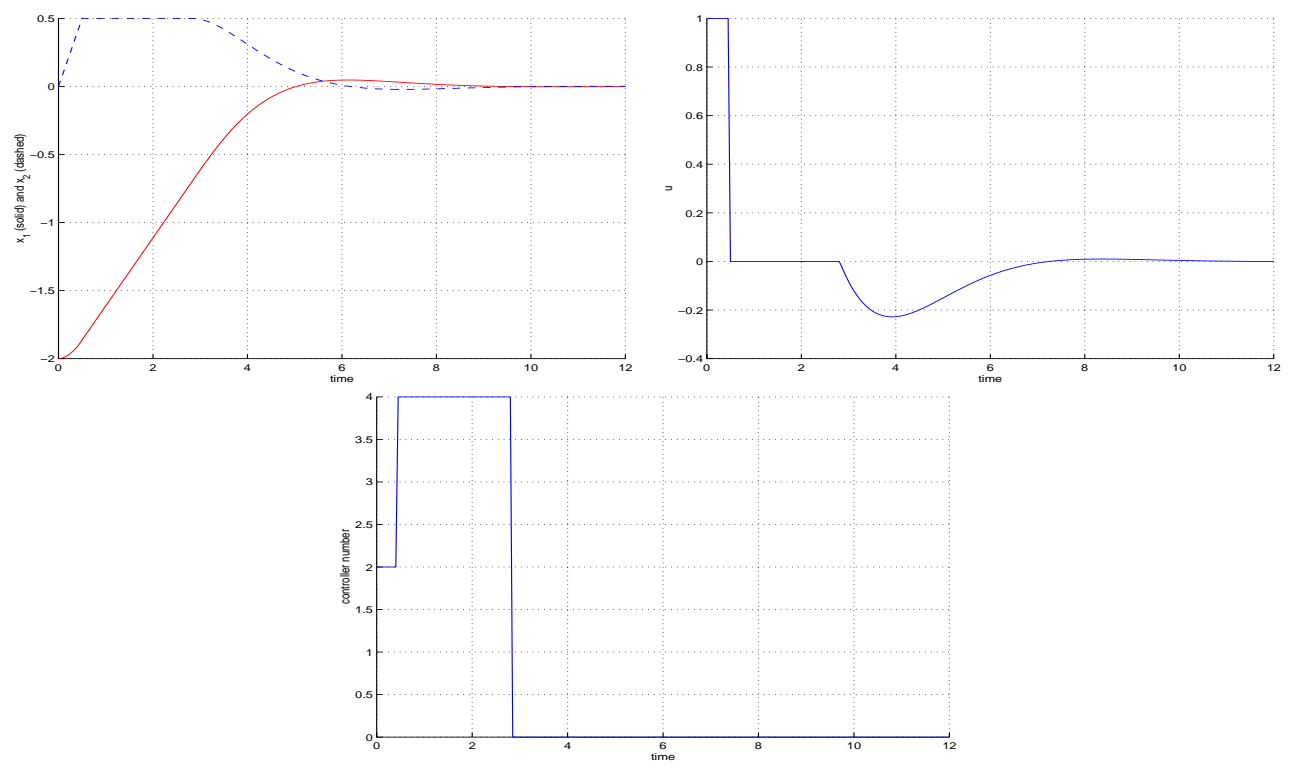

Figure 1: Constrained control of a double integrator from initial state $x(0)=(-2,0)^{T}$.

efficiently handle cases when $\left|x_{2}\right|>0.55$, we define the input constraints (58) as hard (non-relaxable) constraints, and the state constraints (57) as soft (relaxable) constraints. Furthermore, we define all the elements of the weight vector $\omega_{1, \beta_{s o f t}}$ to be equal to one. Since the hard constaints are assoicated with the input only, $X^{R}=R^{2}$. The piecewise linear feedback control law is shown in Figure 2, and the state velocity vector field for the closed loop system

$$
\dot{x}=A_{c} x+B_{c} u^{*}(x, g, h)
$$

is shown in Figure 3. It can be seen that the closed loop system behaves qualitatively as desired consistently over the whole state space. Note in particular that the state constraint $x_{2}=0.5$ is attractive (and active) for sufficiently negative $x_{1}$ and the active state constraint $x_{2}=-0.5$ is attractive (and active) for sufficiently positive $x_{1}$. It can also be seen that the constrained LQ design typically leads to a high gain feedback enforcing the state constraints to be active. This is clearly seen near the state constraints $x_{2}= \pm 0.5$ in the double integrator example in Figures 2 and 3 (it can be verified that that the feedback is indeed continuous with high gain rather than discontinuous, even though this is not apparent from this figure). One can also see that the gain will increase as the sampling interval is reduced, and this high gain may lead to undesirable chattering of the control signal in case of model uncertainty, noise or disturbances.

\subsection{Avoiding high-gain feedback, chattering and sliding modes}

Effectively, the active state constraint is enforced by a sliding-mode like strategy in the example above, see Figures 2 and 3 . This is partly due to the choice of a very small $N$, but the problem can be resolved by modifying the state constraints (10) such that they do not require the active state constraint sets to be fulfilled in a dead-beat manner (at the first possible sample), but rather attract the state asymptotically towards the active constraints. This is achieved by replacing every instant of the active state constraint equation $G_{\beta} x(t+\tau)=g_{\beta}$ by its asymptotic version

$$
G_{\beta} x(t+\tau)=D_{\beta}^{\tau} G_{\beta} x(t)+\left(I-D_{\beta}^{\tau}\right) g_{\beta}
$$

for $\tau \geq 1$. Defining $z(t)=G_{\beta} x(t)-g_{\beta}$, it is clear that (63) implies $z(t+1)=D_{\beta} z(t)$, where the speed of convergence of the state towards the active constraint set can be tuned by the choice of $D_{\beta}$. In order take full advantage of this modification, it is convenient to introduce another modification, namely an $\varepsilon$-boundary layer near each active state constraint, similar to what is common in sliding mode control (Slotine 1984). Within this boundary layer, the controller is only allowed to switch to feedbacks that either makes the associated state constraints asymptotically active, or makes the 


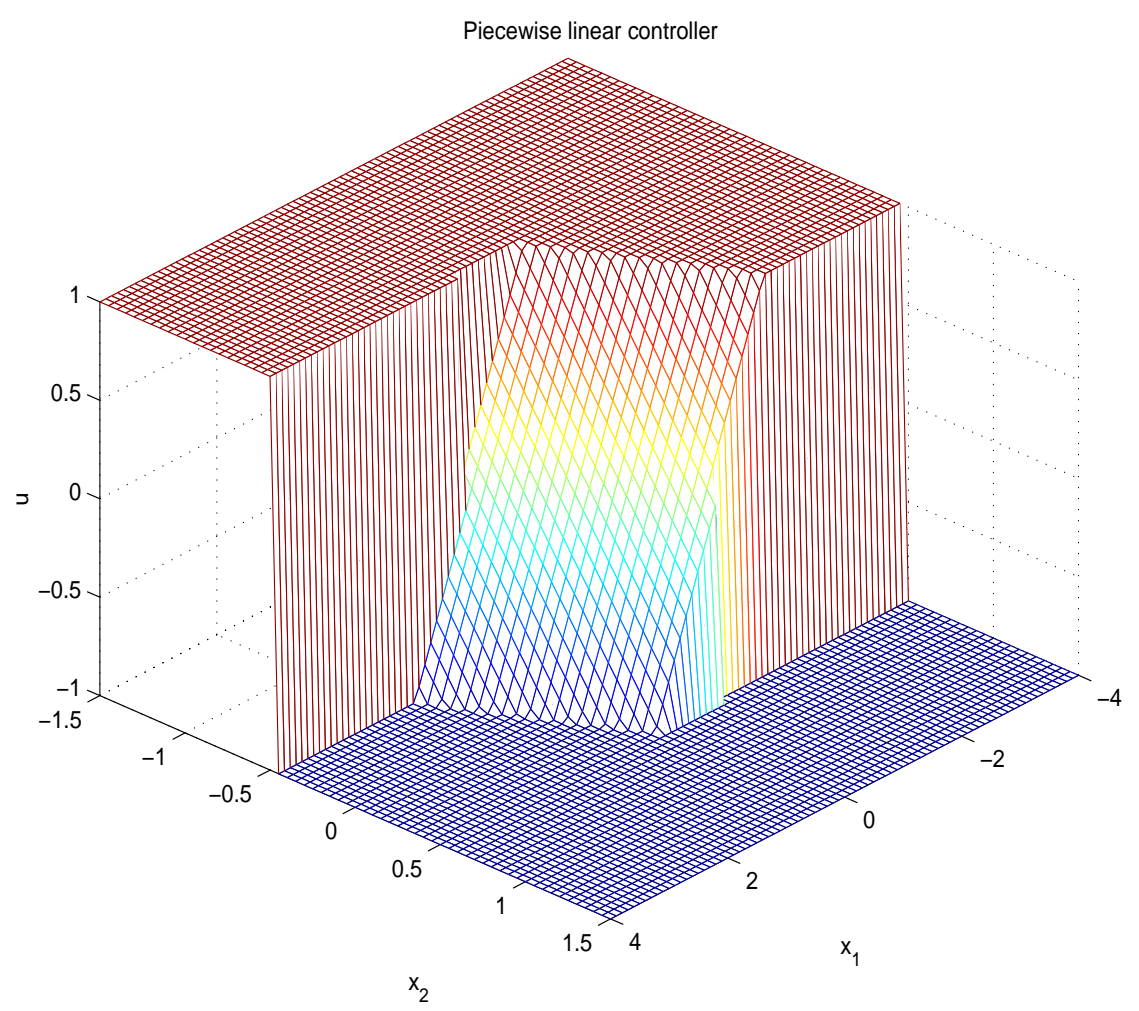

Figure 2: PWL constrained LQ feedback controller for the double integrator.

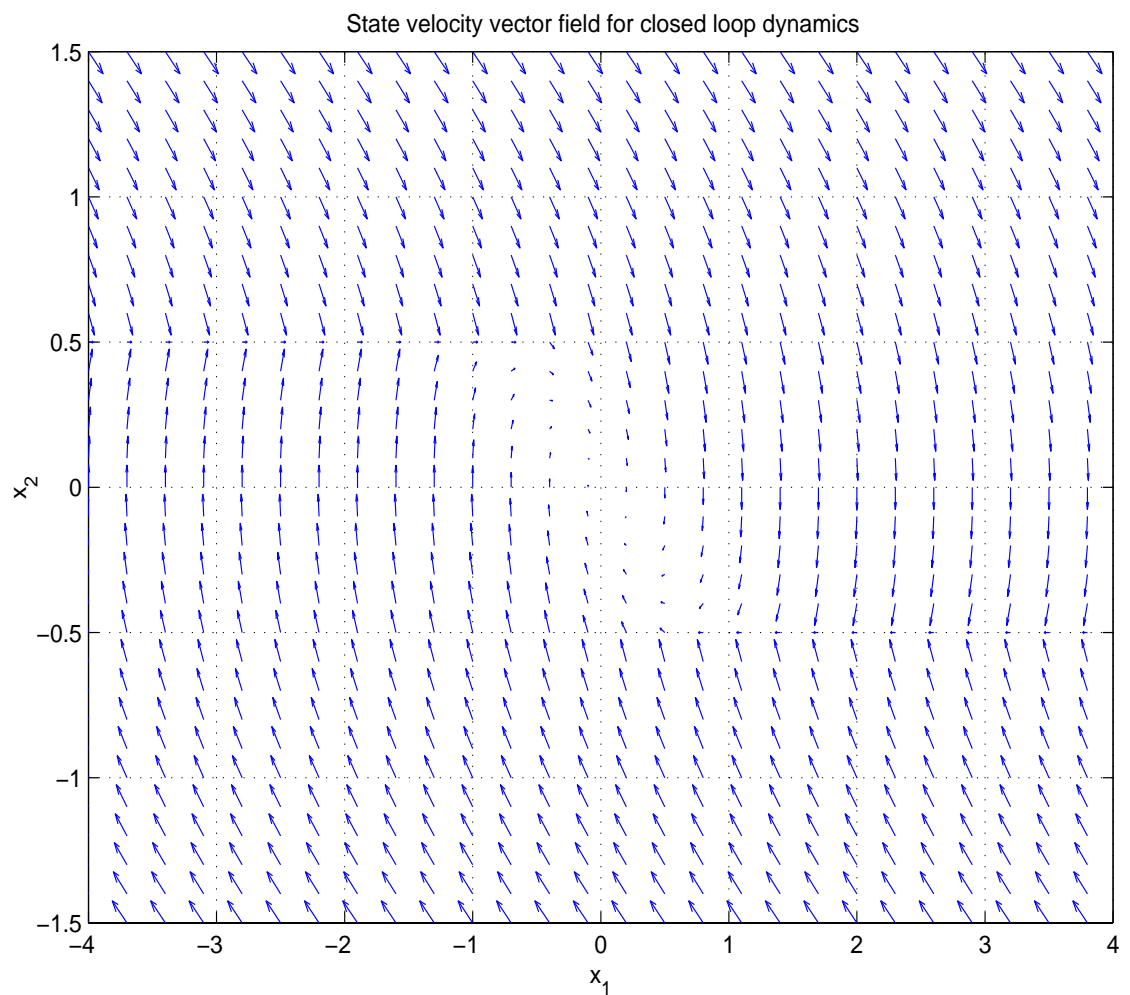

Figure 3: State velocity vector field for the piecewise linear closed loop dynamics consisting of the double integrator with constrained LQR. 
state move away from the state constraint in the direction of the admissible region of the state space. Formally, this is achieved by adding the following constraint to the optimization problem $(24)-(27)$

$$
G_{\beta(x, g)}\left(A^{\tau} x(t)+C_{N} \tilde{E}_{\tau} \tilde{u}_{k}^{*}(x(t), g, h)\right) \leq G_{\beta(x, g)} x(t), \quad \text { if } \beta_{k} \subset \beta(x, g)
$$

for $1 \leq \tau \leq N$. The symbol $\beta(x, g)$ denotes the set of currently $\varepsilon$-active state constraints at $(x, g)$, i.e. $\beta(x, g)=\left\{l \in\{1,2, \ldots, q\}||\left(G_{l 1}, \ldots, G_{l n}\right) x-g_{l} \mid \leq \varepsilon_{l}\right\}$, where $\varepsilon_{l}>0$ defines the boundary layer. Eq. (64) excludes non-attractive feedbacks that tend to move the state towards violation of active state constraints.

\section{Double integrator example, cont'd}

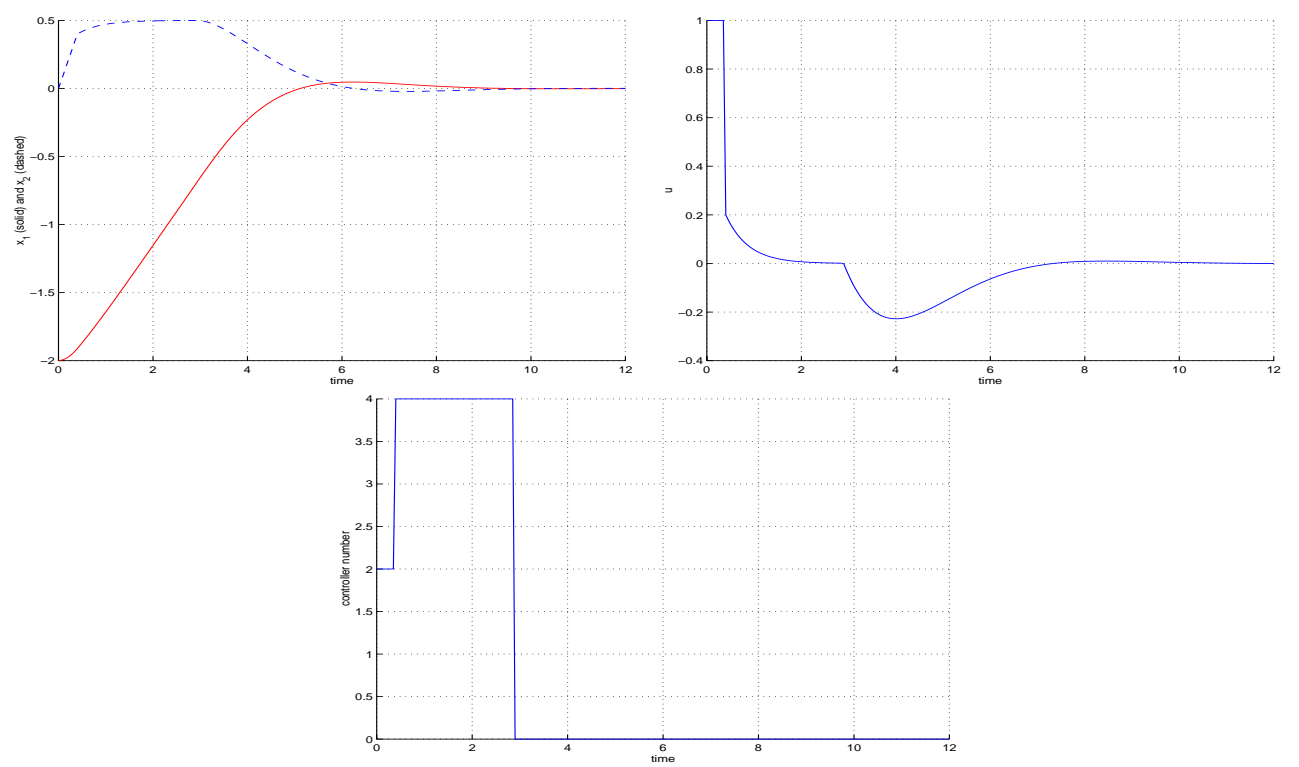

Figure 4: Constrained control of a double integrator from initial state $x(0)=(-2,0)^{T}$, with a boundary layer around the active state constraints.

In order to reduce the gain near the active state constraints, a boundary layer of $\varepsilon_{1}=\varepsilon_{2}= \pm 0.15$ is defined around the constraints $x_{2}=0.5$ and $x_{2}=-0.5$. Hence, when $0.65 \geq x_{2} \geq 0.35$, the control strategy is allowed to switch and the controller takes the objective of attracting the state towards the surface $x_{2}=0.5$ while minimizing the LQ objective. The speed of the motion towards the surface $x_{2}=0.5$ is defined by $D_{1}=D_{2}=0.9$. Simulation results are shown in Figure 4 . Comparing with the original controller without this boundary layer, Figure 1, we observe that the state constraint is now approached only asymptotically. Comparing the piecewise linear controller surface, Figure 5, of the modified controller with the original controller, Figure 2, it is seen that the gain has indeed been reduced in an $\varepsilon$-boundary layer near the active constraints $x_{2}= \pm 0.5$. Also, the state velocity vector plot in Figure 6 shows that the speed of the state is being reduced well before the active state constraint is met. It can be seen that the modified PWL feedback control law in Figure 5 is actually discontinuous at $x_{2}= \pm 0.35$ and $x_{2}= \pm 0.65$, in contrast to the PWL optimal constrained LQ controller (Figure 2) that is proved to be continuous everywhere. However, it is evident from Figure 5 that the discontinuity surface is non-attractive and will not lead to any sliding modes, chattering or other undesirable phenomena with the modified PWL feedback.

Next, we introduce unmodelled dynamics with a transfer function

$$
H(z)=\frac{0.3}{z-0.7}
$$

at the system input. It is clear from the simulation results in Figure 7 that the modified controller indeed eliminates the chattering present in the original controller. 


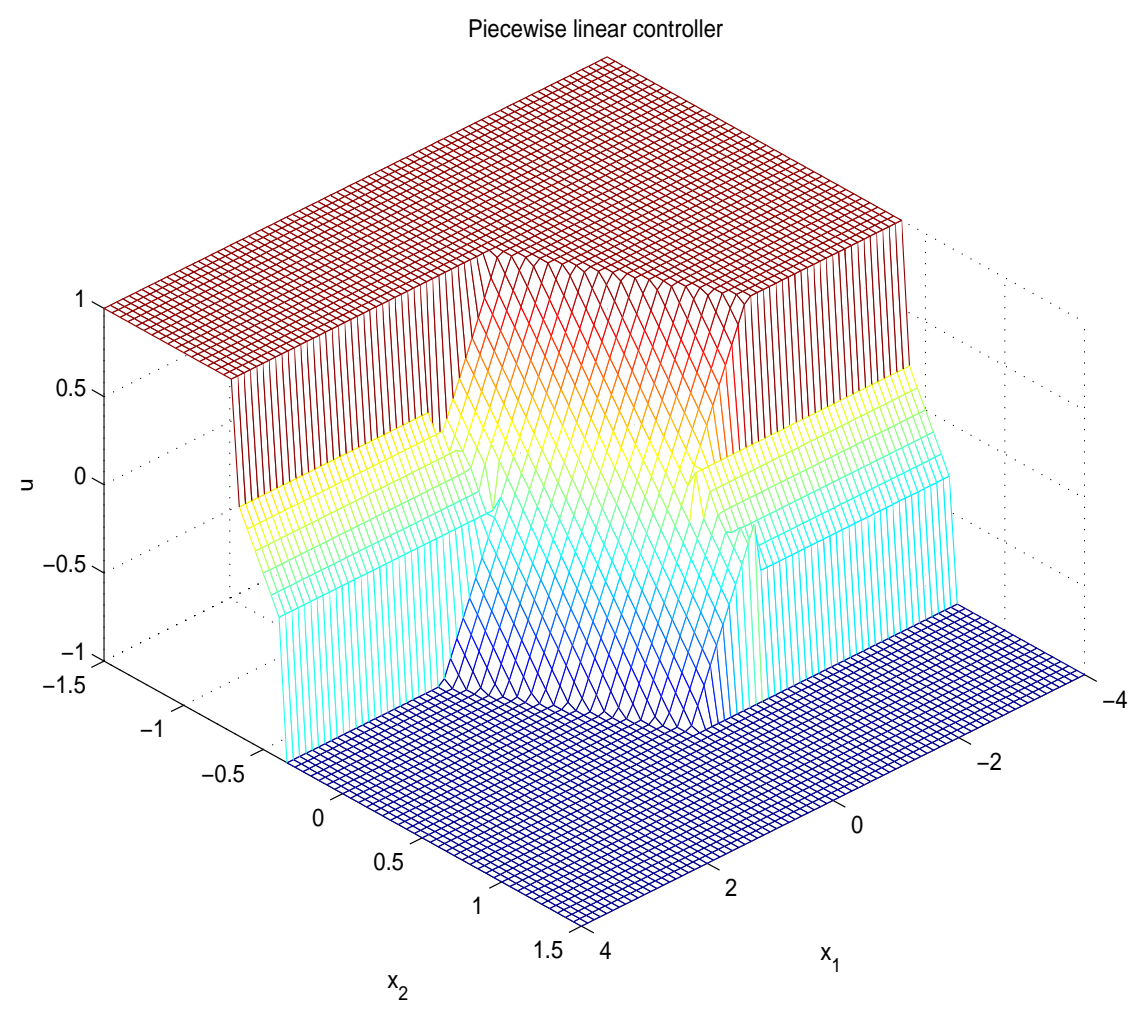

Figure 5: PWL constrained LQ controller for the double integator, with a boundary layer around the active state constraints.

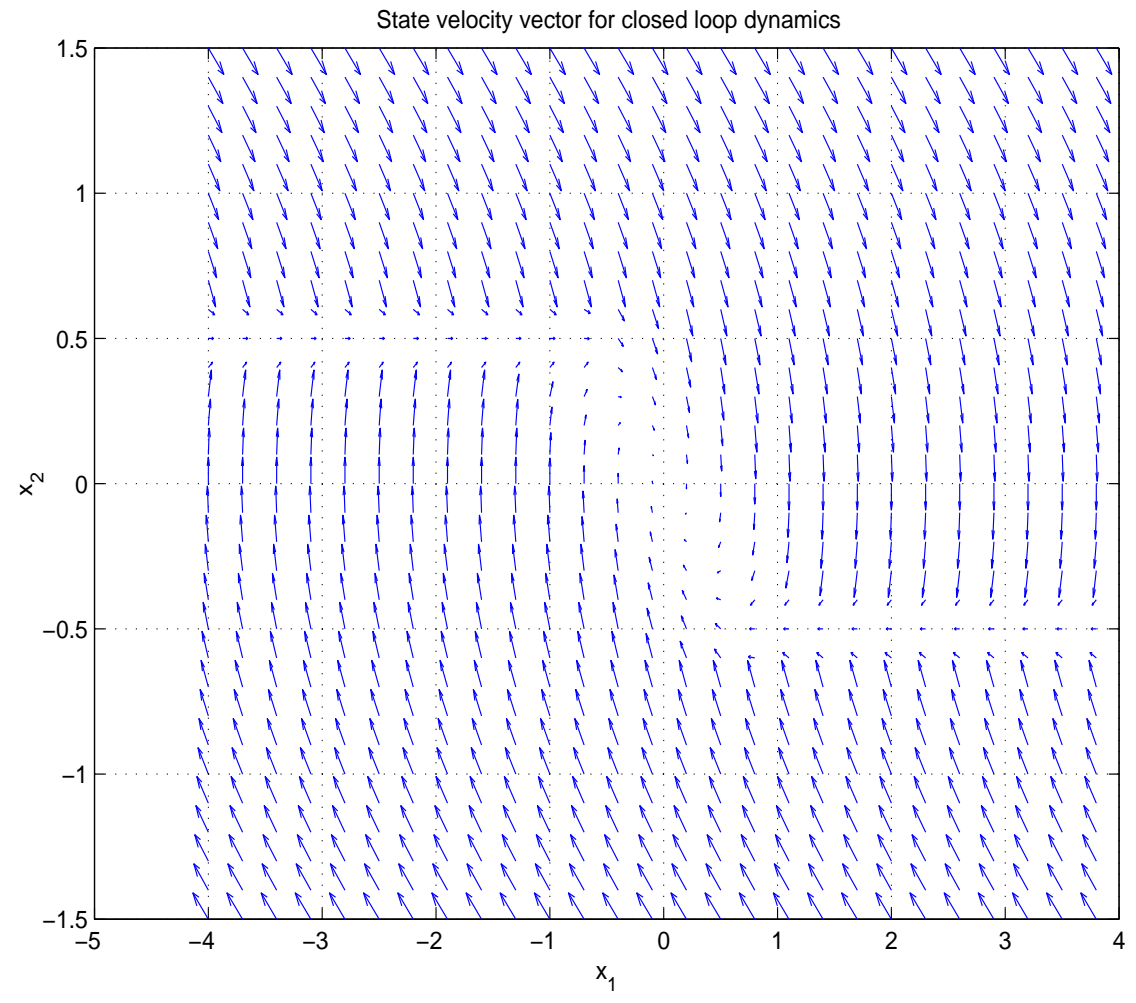

Figure 6: State velocity vector field for the PWL closed loop dynamics of the double integrator with constrained LQR using a boundary layer around the active state constraints. 

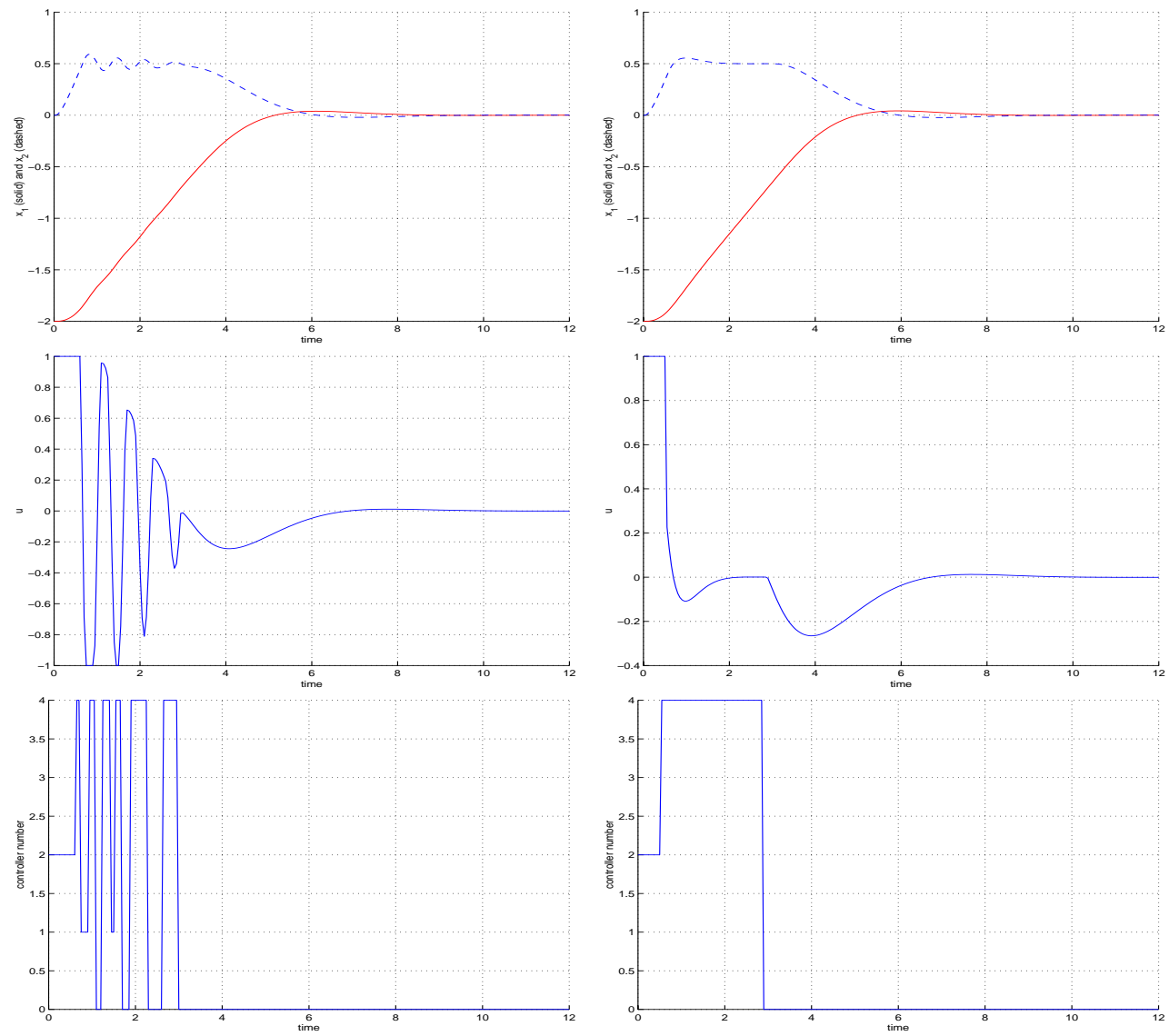

Figure 7: Constrained control of a double integrator from initial state $x(0)=(-2,0)^{T}$, with unmodelled dynamics. The curves in the left column are with the original controller, while the curves in the right columns are with a boundary layer around the active state constraints. 


\subsection{Setpoint tracking}

It has been assumed so far that the setpoint equals the origin. This can be generalized by shifting any equilibrium of the linear system (2) to the origin. Obviously, the constraint limits on both the state and input must be shifted accordingly, but this is straightforward to implement since the precomputed feedback matrices do not depend on the values of the limits.

\section{Real-time Implementation}

The suboptimal constrained LQR is a PWL function of the state. However, efficient evaluation of this PWL function in the real-time control system requires that one is able to efficiently compute in real time which affine feedback to associate with each vector $(x, g, h)$. The affine state feedbacks are computed offline and stored in real-time computer memory. Whether it is desirable to also compute offline an explicit characterization of the subsets of $X \times \mathcal{G} \times \mathcal{H}$ where each affine feedback is active depends on several factors: Acceptable offline processing time, available real-time computer memory and real-time computer processing capacity. Certainly, in a high-dimensional problem with a large number of regions, an explicit offline characterization of the regions where each affine feedback is active is unlikely to be computationally attractive.

There exist at least two real-time implementation strategies that can be employed in order to address the above mentioned tradeoffs:

1. The discrete optimization problems (24)-(27) and (48)-(50) are solved in real time. Discrete search techniques such as branch-and-bound and $A^{*}$ can be applied for this purpose (Korf 1990).

2. A partitioning of $X \times \mathcal{G} \times \mathcal{H}$ such that within each constituent region of the partition there are at most a given small number of affine feedbacks that may be optimal. A search among the small number of remaining candidates (if more than one) is then carried out in real time.

The PWL structure of the controller is explored in Section 4, and a paritioning algorithm, which be used in with the second strategy mentioned above, is described.

\section{Piecewise Linear Structure of the Controller}

The PWL control structure resulting from the design procedure in section 2 may be summarized by the block diagram in Figure 8 . There is a bank of affine feedback controllers of the form $u=K_{k, 2} x+K_{k, 1}^{g} g+K_{k, 1}^{h} h$, where each affine feedback controller is designed with the objective of minimizing the LQ cost function subject to the state and input trajectories moving on a specific active constraint set sequence. In other words, each affine feedback controller will force selected state and input constraints to be active at various samples on the horizon and use the additional available degrees of freedoms (if any) to minimize the LQ objective or minimize the constraint violations if this cannot be avoided. The affine state feedbacks are designed offline, so the real-time computations amount to selecting which affine state feedback to apply at a given state $x(t)$ and computing the control input using the associated precomputed gain matrices $K_{k, 1}^{g}, K_{k, 1}^{h}$ and $K_{k, 2}$. The control structure in Figure 8 corresponds to a hybrid or switching controller since it switches between a number of affine feedbacks, similar to (Johansson and Rantzer 1998, Branicky 1998, Morse 1997).

The purpose of this section is to explore the PWL structure of the suboptimal LQ controller, which can be exploited for at least three purposes:

- One may be able to eliminate certain active constraint set sequences from $\mathcal{C}$ that are never optimal.

- The discrete minimizations in (24) and (48) can either be eliminated completely because the candidate optima may be reduced to single elements within subsets of the state space, or at least be reduced to a small subset of $\mathcal{C}$ within subsets of the state space. This can be exploited 


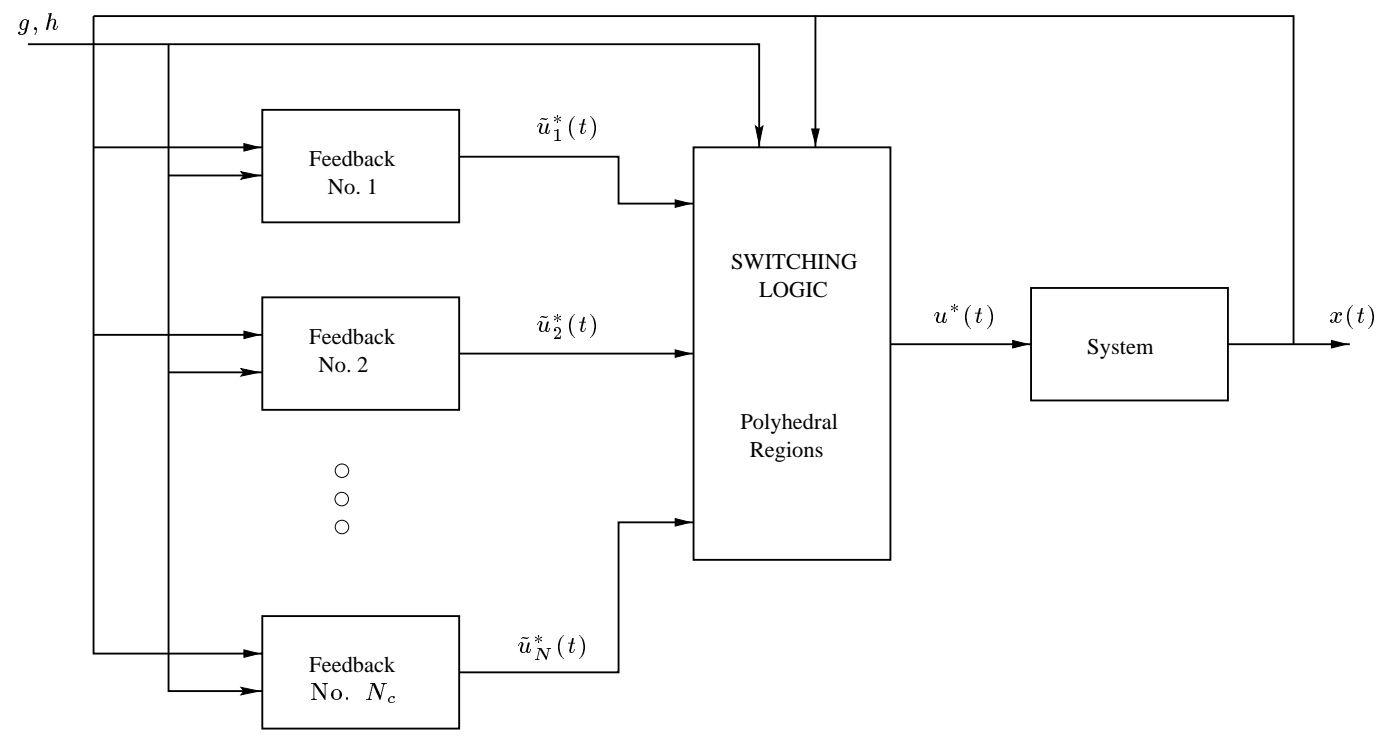

Figure 8: PWL constrained LQR control structure.

in the real-time implementation to reduce the processing capacity requirements as mentioned in section 3 .

- The closed loop performance and stability can be analysed using piecewise linear systems theory and computational tools, see section 5 .

\subsection{Activity regions}

The activity region $Z_{k} \subset Z$ is defined as the subset of the state and constraint limit space where the active constraint set sequence with index $k$ is active, i.e.

$$
Z_{k}=\left\{(x, g, h) \in Z \mid k=k^{*}(x, g, h)\right\}
$$

Together with the affine functions (39), the activity regions $\left\{Z_{k}, k \in \mathcal{C}\right\}$ completely describes the PWL structure of the controller.

\section{Double integrator example, activity regions}

For the double integrator example, there are five constituent affine feedbacks with corresponding activity regions. Since $g=0.5$ and $h=1$ are fixed we only consider the projection of $Z_{k}$ into the state space: $X_{k}(g, h)$. Region/Feedback 0: unconstrained case $(k=0)$, Region/Feedback 1: input constraint $u=-1$ active $(k=1)$, Region/Feedback 2: input constraint $u=1$ active $(k=2)$, Region/Feedback 3 : state constraint $x_{2}=-0.5$ active $(k=3)$, Region/Feedback 4 : state constraint $x_{2}=0.5$ active $(k=4)$. The activity regions for the suboptimal constrained LQ controller with boundary layer are shown in Figure 9. Within each region, the feedback is linear, cf. Figure 5. We observe that in this case the regions can be characterized as unions of polyhedra.

In order to explicitly characterize the activity regions, it is natural to treat the feasible and relaxed feasible regions $Z^{F}$ and $Z^{R}$ separately, since the choice of optimal active constraint set sequence is based on different criteria in these cases. Thus, we define the activity regions contained in $Z^{F}$ as follows:

$$
Z_{k}^{f}=\left\{(x, g, h) \in Z_{k}^{F} \mid k \text { is optimal w.r.t. }(24)-(27) \text { and }(64)\right\}
$$

For $(x, g, h) \in Z^{R}$, the controller objective changes to minimize the constraint violation and we define

$$
Z_{k}^{r}=\left\{(x, g, h) \in Z_{k}^{R} \mid k \text { is optimal w.r.t. }(48)-(50)\right\}
$$




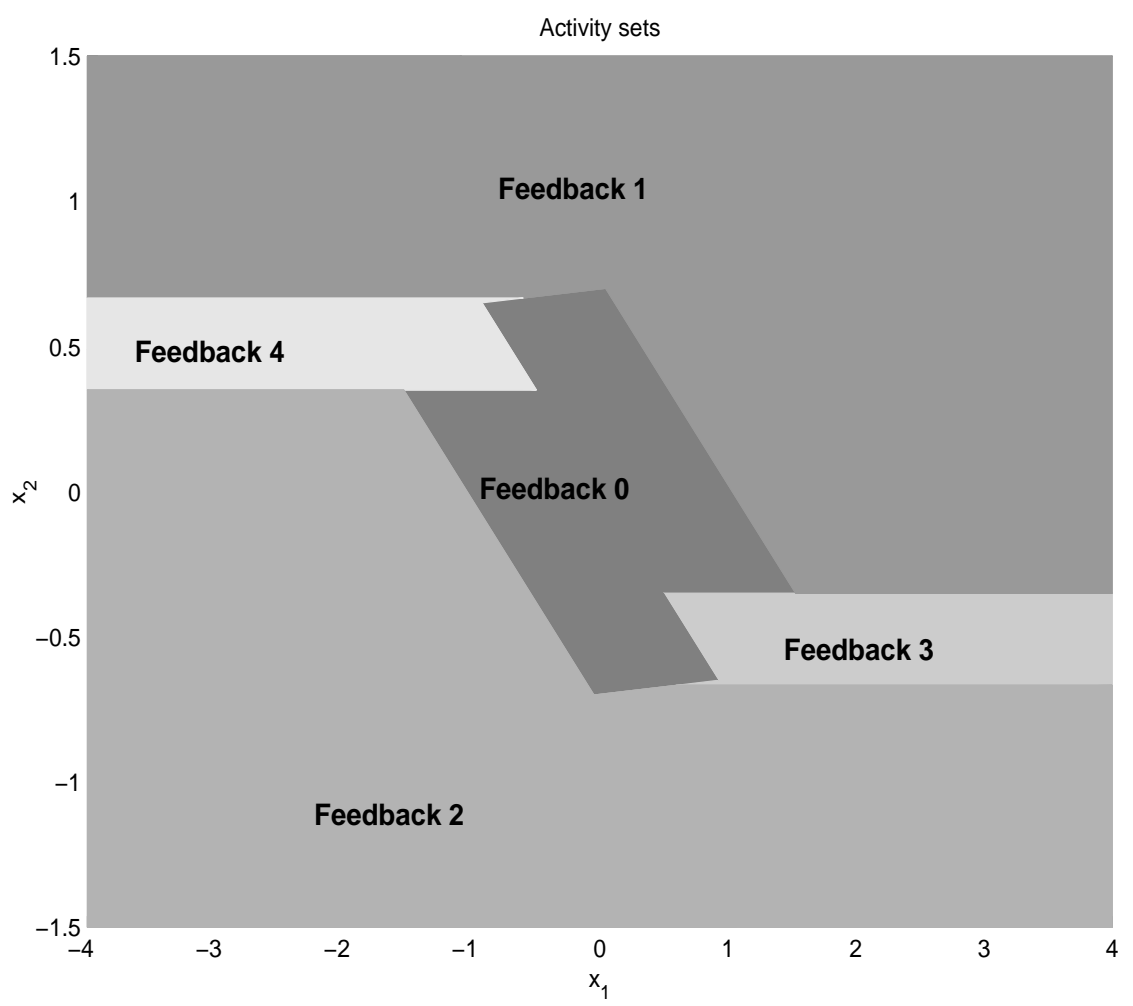

Figure 9: Activity regions for the five constituent affine feedbacks in the constrained LQR for the double integrator with boundary layers.

Hence, the activity region $Z_{k}$ where the feedback with index $k$ is active is now $Z_{k}=Z_{k}^{f} \cup Z_{k}^{r}$. A slightly more explicit characterization of $Z_{k}^{f}$ than (67) is

$$
Z_{k}^{f}=\left\{(x, g, h) \in Z_{k}^{F} \mid \varphi_{k}(x, g, h) \leq \varphi_{j}(x, g, h), \text { for all } j \in \mathcal{F}(x, g, h) \cap \mathcal{A}(x, g, h)\right\}
$$

where $\mathcal{F}(x, g, h) \subset \mathcal{C}$ is a set containing the active constraint set sequences that are feasible at $x$

$$
\mathcal{F}(x, g, h)=\left\{k \in \mathcal{C} \mid(x, g, h) \in Z_{k}^{F}\right\}
$$

and $\mathcal{A}(x, g, h) \subset \mathcal{C}$ is a set containing the indices to the active constraint set sequences that are attractive or not currently active at $(x, g, h)$, cf. (64):

$$
\mathcal{A}(x, g, h)=\left\{k \in \mathcal{C} \mid G_{\beta(x, g)}\left(A^{\tau} x+C_{N} \tilde{E}_{\tau} \tilde{u}_{k}^{*}(x, g, h)\right) \leq G_{\beta(x, g)} x \text { or } \beta_{k} \not \subset \beta(x, g)\right\}
$$

Furthermore, since there exists an optimal solution if and only if there exists a feasible solution to (24)-(27) it follows that

$$
Z^{F}=\bigcup_{k \in \mathcal{C}} Z_{k}^{f}=\bigcup_{k \in \mathcal{C}} Z_{k}^{F}
$$

Likewise, a slightly more explicit characterization of $Z_{k}^{r}$ than (68) is given by

$$
Z_{k}^{r}=\left\{(x, g, h) \in Z_{k}^{R} \mid \nu_{k}(x, g, h) \leq \nu_{j}(x, g, h), \text { for all } j \in \mathcal{R}(x, g, h)\right\}
$$

where $\mathcal{R}(x, g, h) \subset \mathcal{C}$ is defined as the set of active constraint set sequences that are feasible with respect to the non-relaxable constraints, but not feasible with respect to the relaxable constraints, at $(x, g, h)$ :

$$
\mathcal{R}(x, g, h)=\left\{k \in \mathcal{C} \mid(x, g, h) \in Z_{k}^{R}\right\}
$$

and we also have

$$
Z^{R}=\bigcup_{k \in \mathcal{C}} Z_{k}^{r}=\bigcup_{k \in \mathcal{C}} Z_{k}^{R}
$$


which is the set of states where there exists an active constraint set sequence that is feasible and optimal with respect to the non-relaxable constraints but not with respect to the relaxable constraints.

\subsection{Outer Approximations to the Activity Regions}

Since $Z_{k}^{F}$ and $Z_{k}^{R}$ are polyhedral, it is clear that $Z^{F}, Z^{R}$ and $Z=Z^{F} \cup Z^{R}$ are unions of polyhedra. However, beause the optimality conditions in (69) are characterized by quadratic functions, the set $Z_{k} \subset Z$ may not be characterized only by the hyperplanes defined by feasibility, but possibly also by other hyperplanes or (convex or nonconvex) quadratic surfaces due to the optimality conditions. Thus, $Z_{k}$ may in general not be a union of polyhedra and therefore difficult to characterize exactly in a more explicit manner than (69) and (73). Still, several explicit outer approximations of $Z_{k}$ can be computed in terms of sets that contain $Z_{k}$. These are useful both for real-time implementation (2nd strategy in Section 3), and for computational analysis, Section 5.

Here we develop an outer approximation $\bar{Z}_{k} \supset Z_{k}$ where $\bar{Z}_{k}$ is a union of polyhedra. As the basic polyhedral building blocks in this characterization we consider the hyperplane partition $\mathcal{P}_{Z}^{H P}=$ $\left\{\mathcal{Z}_{l}^{H P} \mid l \in\left\{1,2, \ldots, N_{P}\right\}\right\}$ generated by all the hyperplanes involved in the characterization of $Z_{k}^{F}, Z_{k}^{R}$ and $Z_{k}^{A}$, for $k \in \mathcal{C}$ :

$$
\begin{aligned}
H E_{\tau} K_{k, 2} x & =h-H E_{\tau}\left(K_{k, 1}^{g} g+K_{1, k}^{h} h\right), & & \text { Input constraints } \\
G\left(A^{\tau}+C_{N} \tilde{E}_{\tau} K_{k, 2}\right) x & =g-G C_{N} \tilde{E}_{\tau}\left(K_{k, 1}^{g} g+K_{k, 1}^{h} h\right), & & \text { State constraints } \\
G\left(A^{\tau}+C_{N} \tilde{E}_{\tau} K_{2, k}-I\right) x & =G C_{N} \tilde{E}_{\tau}\left(K_{1, k}^{h} h+K_{1, k}^{g} g\right), & & \text { Attractivity }
\end{aligned}
$$

for $1 \leq \tau \leq N$. Let (76)-(78) be written in compact notation $Y z=y$ where $z=(x, g, h)$ and $Z \in R^{N_{z} \times(n+q+p)}, z \in R^{N_{z}}$ and $N_{z}$ is the number of equations in (76)-(78). The set of halfspaces $\mathcal{Y}_{i}^{+}=\left\{z \in R^{n+q+p} \mid Y_{i} z \geq y_{i}\right\}$ and $\mathcal{Y}_{i}^{-}=\left\{z \in R^{n+q+p} \mid Y_{i} z<y_{i}\right\}$ now defines the hyperplane partition $\mathcal{P}_{Z}^{H P}$ of $Z$ as the set of all possible non-empty intersections of halfspaces: $\mathcal{Z}_{l}^{H P}=\mathcal{Y}_{1}^{*} \cap$ $\ldots \cap \mathcal{Y}_{N_{z}}^{*}$ where $*$ symbolizes any combinations of $+/-$. Note that this hyperplane partition will contain unnecessarily many elements in many cases and is introduced here in order to develop a theoretical understanding. Computational algorithms should as far as possible operate on a coarser partition.

\section{Double integrator example, hyperplane partition}

The hyperplanes and associated polyhedra defining the regions of feasibility for the constrained LQR with boundary layer for the double integrator are shown in Figure 10. In total, there are 20 hyperplanes leading to 78 polyhedra in this hyperplane partition. Comparing the hyperplane partition in Figure 10 with the activity sets in Figure 9, it is clear that the polyhedra constituting the hyperplane partition can be used to characterize the unions of polyhedra defining the activity regions.

Lemma 2 The hyperplane partition $\mathcal{P}_{Z}^{H P}$ has the following properties:

1. Each constituent region of the partition is uniquely associcated with either the feasible region $Z^{F}$ or the relaxed feasible region $Z^{R}$, i.e. $\mathcal{Z}_{l}^{H P} \cap Z^{F}=\emptyset$ and $\mathcal{Z}_{l}^{H P} \cap Z^{R}=\mathcal{Z}_{l}^{H P}$, or vice versa $\mathcal{Z}_{l}^{H P} \cap Z^{F}=\mathcal{Z}_{l}^{H P}$ and $\mathcal{Z}_{l}^{H P} \cap Z^{R}=\emptyset$, for all $l=1,2, \ldots ., N_{P}$.

2. For all $l \in\left\{1,2, \ldots, N_{p}\right\}$ and $(x, g, h) \in \mathcal{Z}_{l}^{H P}$ the sets $\mathcal{F}(x, g, h), \mathcal{R}(x, g, h), \mathcal{A}(x, g, h)$ and $\beta(x, g)$ are invariant in the sense that each of them contain the same elements for all $(x, g, h) \in$ $\mathcal{Z}_{l}^{H P}$.

Proof. Follows from the fact that the hyperplane partition $\mathcal{P}_{Z}^{H P}$ of $Z$ is generated by all hyperplanes involved in the characterizations of $\mathcal{F}(x, g, h), \mathcal{R}(x, g, h), \mathcal{A}(x, g, h)$ and $\beta(x, g)$. 


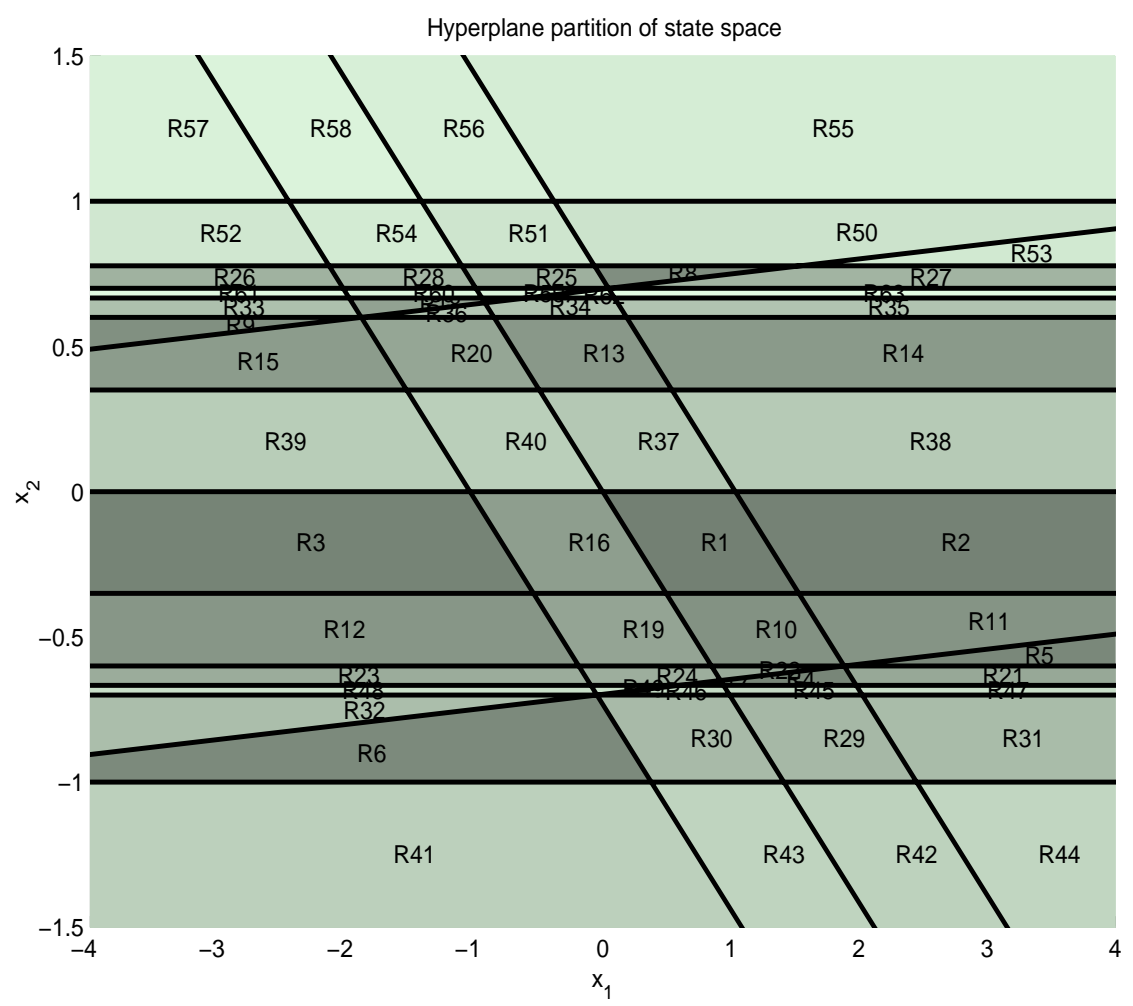

Figure 10: Hyperplane partition for the constrained LQR for the double integrator with boundary layers.

From the first part of Lemma 2 it is evident that each $\mathcal{Z}_{l}^{H P} \in \mathcal{P}_{Z}^{H P}$ is fully contained in either $Z^{R}$ or $Z^{F}$. Thus, we define disjoint index sets

$$
\begin{aligned}
\mathcal{L}^{F} & =\left\{l \in\left\{1,2, \ldots, N_{P}\right\} \mid \mathcal{Z}_{l}^{H P} \cap Z^{F} \neq \emptyset\right\} \\
\mathcal{L}^{R} & =\left\{l \in\left\{1,2, \ldots, N_{P}\right\} \mid \mathcal{Z}_{l}^{H P} \cap Z^{R} \neq \emptyset\right\}
\end{aligned}
$$

Assume $l \in \mathcal{L}^{F}$, i.e. $\mathcal{Z}_{l}^{H P} \subset Z^{F}$. One may now define a set $\mathcal{F}_{l}^{f} \subset \mathcal{C}$ of feasible active constraint set sequences in the region $\mathcal{Z}_{l}^{H P}$ :

$$
\mathcal{F}_{l}^{f}=\left\{k \in \mathcal{C} \mid \mathcal{Z}_{l}^{H P} \cap Z_{k}^{F} \neq \emptyset\right\}
$$

Hence, for any $(x, g, h) \in Z^{F}$ there exists a unique $l(x, g, h) \in \mathcal{L}^{F}$ such that $(x, g, h) \in Z_{l(x, g, h)}^{F}$ and at least one of the feasible active constraint set sequences in $\mathcal{F}_{l}^{f}$ is optimal for all $(x, g, h) \in \mathcal{Z}_{l}^{H P}$. We continue by characterizing the subset of $\mathcal{F}_{l}^{f}$ that is optimal for some $(x, g, h) \in \mathcal{Z}_{l}^{F}$, aiming towards a definition of $\bar{Z}_{k}^{f} \supset Z_{k}^{f}$.

Lemma 3 Let $l \in \mathcal{L}^{F}$ and $j, k \in \mathcal{F}_{l}^{f}$ be arbitrary. Suppose the active constraint set sequences $\left(\left(\alpha_{1}^{k}, \beta_{1}^{k}\right),\left(\alpha_{2}^{k}, \beta_{2}^{k}\right), \ldots,\left(\alpha_{N_{S}}^{k}, \beta_{N_{S}}^{k}\right)\right)$ and $\left(\left(\alpha_{1}^{j}, \beta_{1}^{j}\right),\left(\alpha_{2}^{j}, \beta_{2}^{j}\right), \ldots,\left(\alpha_{N_{S}}^{j}, \beta_{N_{S}}^{j}\right)\right)$ are different. If $\left(\alpha_{i}^{k}, \beta_{i}^{k}\right) \subset$ $\left(\alpha_{i}^{j}, \beta_{i}^{j}\right)$, for all $i=1,2, \ldots, N_{S}$, then the active constraint set sequence with index $j$ is suboptimal for all $(x, g, h) \in \mathcal{Z}_{l}^{H P}$.

Proof. Because the active constraint set sequence with index $k$ is a subset of the active constraint set sequence with index $j$ and both are feasible, it follows immediately that $\varphi_{k}(x, g, h) \leq \varphi_{j}(x, g, h)$ for all $(x, g, h) \in \mathcal{Z}_{l}^{H P}$ since adding a constraint to some constraint set sequence will not reduce the cost. 
Lemma 4 Let $l \in \mathcal{L}^{F}$ and $k \in \mathcal{F}_{l}^{f}$ be arbitrary, and define

$$
\begin{aligned}
& \gamma_{j k}=\max _{(x, g, h) \in \mathcal{Z}_{l}^{H P}}\left(\varphi_{k}(x, g, h)-\varphi_{j}(x, g, h)\right) \\
& \kappa_{j k}=\min _{(x, g, h) \in \mathcal{Z}_{l}^{H P}}\left(\varphi_{k}(x, g, h)-\varphi_{j}(x, g, h)\right)
\end{aligned}
$$

If $\gamma_{j k} \leq 0$ for all $j \in \mathcal{F}_{l}^{f}$, then the active constraint set sequence with index $k$ is optimal for all $(x, g, h) \in \mathcal{Z}_{l}^{H P}$. If $\kappa_{j k} \geq 0$ for all $j \in \mathcal{F}_{l}^{f}$, then the active constraint set sequence with index $k$ is suboptimal for all $(x, g, h) \in \mathcal{Z}_{l}^{H P}$.

Proof. Since $\gamma_{j k} \leq 0$ it follows that for all $(x, g, h) \in \mathcal{Z}_{l}^{H P}$ and $j \in \mathcal{F}_{l}^{f}, \varphi_{k}(x, g, h) \leq \varphi_{j}(x, g, h)$. Note that due to Lemma $2, \mathcal{F}_{l}^{f}=\mathcal{F}(x, g, h)$ for all $(x, g, h) \in \mathcal{Z}_{l}^{H P}$, and the first part follows because $\mathcal{F}_{l}^{f}$ contains all feasible active constraint set sequences in $\mathcal{C}$. The second part of the lemma is analogous.

Note that both (82) and (83) are quadratic programs, for a fixed $l \in \mathcal{L}^{F}$ and fixed active constraint set sequences $k, j \in \mathcal{F}_{l}^{f}$, since $\mathcal{Z}_{l}^{H P}, \mathcal{G}, H$ are polyhedral and $\varphi_{k}$ and $\varphi_{j}$ are quadratic. Note that even though $\varphi_{k}$ and $\varphi_{j}$ are in general convex, their difference may be neither convex nor concave.

Using the optimality characterizations in Lemmas 3 and 4, one will typically be able to exclude a large set of candidate active constraint set sequences from the set of feasible active constraint set sequences $\mathcal{F}_{l}^{f}$ in the region $\mathcal{Z}_{l}^{H P}$. We define $\mathcal{O}_{l}^{f} \subset \mathcal{F}_{l}^{F}$ as the indices of those active constraint set sequences that are consistent with the optimality conditions in Lemmas 3 and 4 in $\mathcal{Z}_{l}^{H P}$ :

$$
\mathcal{O}_{l}^{f}=\left\{k \in \mathcal{F}_{l}^{f} \mid k \text { is optimal w.r.t. (24)-(27), (64) for some }(x, g, h) \in \mathcal{Z}_{l}^{H P}\right\}
$$

Finally, we define the outer approximation to the activity region $Z_{k}^{f}$ as follows:

$$
\bar{Z}_{k}^{f}=\bigcup_{l \in \mathcal{L}^{F}} \mathcal{Z}_{l}^{H P}
$$

Now, assume $l \in \mathcal{L}^{R}$, i.e. $\mathcal{Z}_{l}^{H P} \subset Z^{R}$. One may now define a set $\mathcal{F}_{l}^{r} \subset \mathcal{C}$ of relaxed feasible active constraint set sequences in the region $\mathcal{Z}_{l}^{H P}$ :

$$
\mathcal{F}_{l}^{r}=\left\{k \in \mathcal{C} \mid \mathcal{Z}_{l}^{H P} \cap Z_{k}^{R} \neq \emptyset\right\}
$$

Unlike the characterization of $Z_{k}^{f}$, we now have the following result:

Lemma 5 For all $k \in \mathcal{C}$, the set $Z_{k}^{r}$ is a union of polyhedra.

Proof. Let $k \in \mathcal{C}$ be arbitrary. The region of relaxed feasibility $Z_{k}^{R}$ is polyhedral, cf. (52). Since the function $\nu_{k}(x, g, h)-\nu_{j}(x, g, h)$ is piecewise linear in $(x, g, h)$, the sets $\left\{(x, g, h) \in R^{n} \times \mathcal{G} \times\right.$ $\left.\mathcal{H} \mid \nu_{k}(x, g, h) \leq \nu_{j}(x, g, h)\right\}$ that appear in the optimality condition in (73) are characterized using hyperplanes. Since all geometric objects characterizing $Z_{k}^{r}$ are hyperplanes, it is a union of polyhedral sets.

According to Lemma 5 it is possible to obtain an exact and explicit characterization of $Z_{k}^{r}$. However, for computational reasons it may be convenient with an outer approximation $\bar{Z}_{k}^{r} \supset Z_{k}^{r}$ in some cases. The following optimality lemma is useful in that respect:

Lemma 6 Let $l \in \mathcal{L}^{R}$ and $k \in \mathcal{F}_{l}^{r}$ be arbitrary, and define

$$
\begin{aligned}
& \rho_{j k}=\max _{(x, g, h) \in \mathcal{Z}_{l}^{H P}}\left(\nu_{k}(x, g, h)-\nu_{j}(x, g, h)\right) \\
& \sigma_{j k}=\min _{(x, g, h) \in \mathcal{Z}_{l}^{H P}}\left(\nu_{k}(x, g, h)-\nu_{j}(x, g, h)\right)
\end{aligned}
$$

If $\rho_{j k} \leq 0$ for all $j \in \mathcal{F}_{l}^{r}$, then the active constraint set sequence with index $k$ is optimal for all $(x, g, h) \in \mathcal{Z}_{l}^{H P}$. If $\sigma_{j k} \geq 0$ for all $j \in \mathcal{F}_{l}^{R}$, then the active constraint set sequence with index $k$ is suboptimal for all $(x, g, h) \in \mathcal{Z}_{l}^{H P}$. 
Note that (87) and (88) are (possibly nonconvex) piecewise linear programs. Using the optimality characterizations in Lemma 6 , one will typically be able to exclude a large set of candidate active constraint set sequences from the set of feasible active constraint set sequences $\mathcal{F}_{l}^{r}$ in the region $\mathcal{Z}_{l}^{H P}$. We define $\mathcal{O}_{l}^{r} \subset \mathcal{F}_{l}^{R}$ as the indices of those active constraint set sequences that are consistent with the optimality conditions in Lemma 6 in $\mathcal{Z}_{l}^{H P}$ :

$$
\mathcal{O}_{l}^{r}=\left\{k \in \mathcal{F}_{l}^{r} \mid k \text { is optimal w.r.t. (48)-(50) for some }(x, g, h) \in \mathcal{Z}_{l}^{H P}\right\}
$$

Finally, we define the outer approximation to the activity region $Z_{k}^{r}$ as follows:

$$
\bar{Z}_{k}^{r}=\bigcup_{l \in \mathcal{L}^{R}} \mathcal{Z}_{l}^{H P}
$$

We are now in position to define

$$
\begin{aligned}
\mathcal{F}_{l} & = \begin{cases}\mathcal{F}_{l}^{f}, & l \in \mathcal{L}^{F} \\
\mathcal{F}_{l}^{r}, & l \in \mathcal{L}^{R}\end{cases} \\
\mathcal{O}_{l} & = \begin{cases}\mathcal{O}_{l}^{f}, & l \in \mathcal{L}^{F} \\
\mathcal{O}_{l}^{r}, & l \in \mathcal{L}^{R}\end{cases} \\
\bar{Z}_{k} & =\bar{Z}_{k}^{f} \cup \bar{Z}_{k}^{r}
\end{aligned}
$$

\subsection{Partitioning Algorithm}

The above sets can in principle be computed directly by first determining the hyperplane partition $\mathcal{P}_{Z}^{H P}$ and then using Lemmas 3-6 to compute the candidate optimal affine feedbacks within each region of the partition. However, this procedure may be too computationally intensive for large problems, and a computationally efficient alternative algorithm is required.

Algorithm 1 (Partitioning algorithm)

1. Let $\mathcal{E}:=\emptyset$, and $\mathcal{U}:=\{Z\}$.

2. If $\mathcal{U}=\emptyset$, the partition generated by this algorithm is $\mathcal{P}=\mathcal{E}$ and the algorithm terminates.

3. Let $\mathcal{Z}_{0} \in \mathcal{U}$ be arbitrary.

4. Let $\mathcal{O}_{0}$ contain the candidate optimal active constraint set seqeuences in $\mathcal{Z}_{0}$, computed according to Lemmas 3-6.

5. If $\mathcal{O}_{0}$ contains a sufficiently small number of elements, add $\mathcal{Z}_{0}$ to the set of explored subsets $\mathcal{E}$ and remove $\mathcal{Z}_{0}$ from the set of unexplored subsets $\mathcal{U}$. Go to step 2.

6. Select a hyperplane $Y_{i} z=y_{i}$ from $Y z=y$ and split $\mathcal{Z}_{0}$ into non-empty $\mathcal{Z}_{0}^{+}=\mathcal{Z}_{0} \cap \mathcal{Y}_{i}^{+}$and $\mathcal{Z}_{0}^{-}=\mathcal{Z}_{0} \cap \mathcal{Y}_{i}^{-}$. If this is not possible for any hyperplane from $Y z=y$, add $\mathcal{Z}_{0}$ to the set of explored subsets $\mathcal{E}$ and remove $\mathcal{Z}_{0}$ from the set of unexplored subsets $\mathcal{U}$. Go to step 2.

7. Add $\mathcal{Z}_{0}^{+}$and $\mathcal{Z}_{0}^{-}$to $\mathcal{U}$ and remove $\mathcal{Z}_{0}$ from $\mathcal{U}$. Go to step 2.

The set $\mathcal{E}$ contains the set of explored subsets of $Z$, while the set $\mathcal{U}$ contains the set of explored subsets of $Z$. The algorithm will explore the canidate optimal active constraint sets associated with each element of $\mathcal{E}$ sequentially. The regions of $Z$ will be split using the hyperplanes from $Y z=y$ and explored individually until either a sufficiently small number of candidate optimal active constraint set remains in each region, or the region can not be split any further using hyperplanes from $Y z=y$. The following theorem summarizes the properties of the result of Algorithm 1.

Theorem 3 (Properties of Partitioning Algorithm) Algorithm 1 terminates with a partition $\mathcal{P}_{Z}$ and sets $\mathcal{O}_{l}, \bar{Z}_{k}$ that satisfies 
- $k^{*}(x, g, h) \in \mathcal{O}_{l}$, for all $(x, g, h) \in Z$.

- $Z_{k} \subset \bar{Z}_{k}$, and $\bar{Z}_{k}$ is a union of polyhedra.

In order to reduce the computational complexity of Algorithm 1 one should implement heuristics in step 6 in order to select a "promising" hyperplane for splitting the region $\mathcal{Z}_{0}$ such that unnecessary splitting is avoided. Possibly, the number of candiadate hyperplanes should be restricted in order to keep the offline computational complexity low. However, a detailed discussion of efficient implementation of Algorithm 1 is outside the scope of this paper.

Note that the partition $\mathcal{P}_{Z}$ generated by Algorithm 1 may be unnecessarily fine since at each step it is not known a priori if one can reduce the number of elements in $\mathcal{O}_{0}$ by further partitioning of $\mathcal{Z}_{0}$. This is perhaps not known until $\mathcal{Z}_{0}$ is broken down to its hyperplane partition. Hence, after the algorithm terminates, the number of constituent polyhedra in the partition of $Z$ can often be reduced considerably by aggregating pairs of neighbouring polyhedra whenever their union remains polyhedral.

\section{Double integrator example, cont'd}

The partition computed using the above Algorithm 1 with a successive aggregration of neighbouring regions is shown in Figure 11. We observe the the number of regions is 11, which is the smallest possible number of polyhedral regions capable of characterizing the activity sets for this problem. Also, we observe that within each region, there is a single candidate optimal constraint set. Hence, the PWL feedback law is explicitly characterized by this partition. Feedback 0 (unconstrained case) is associated with R1, R2 and R4 in this partition. Feedback $1(u=-1)$ is associated with R5 and R11. Feedback $2(u=1)$ is associated with R7 and R10. Feedback $3\left(x_{2}=-0.5\right)$ is associated with R2 and R6, while feedback $4\left(x_{2}=0.5\right)$ is associated with R8 and R9.

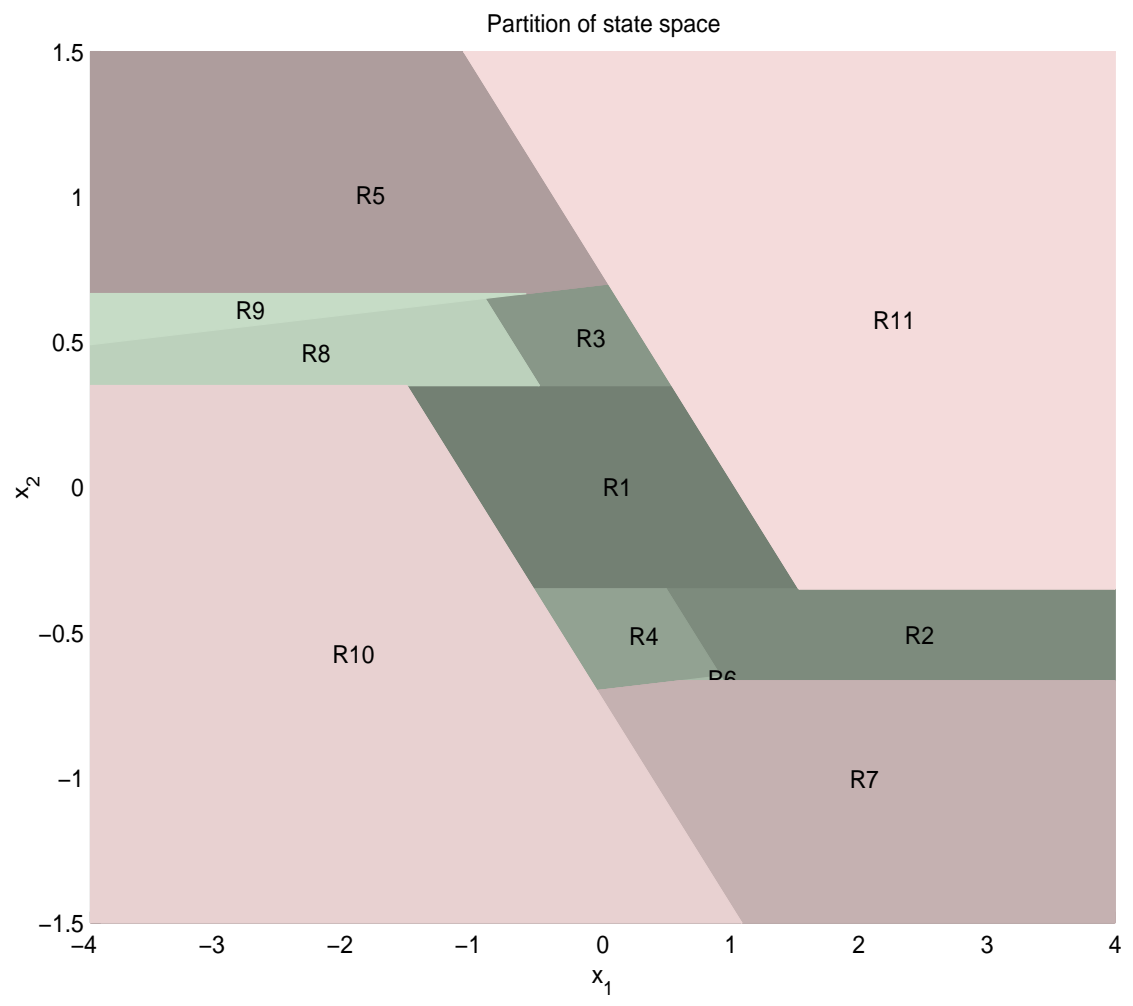

Figure 11: Partition for the constrained LQR for the double integrator with boundary layers.

So far the partitioning has been restricted to utilize only the hyperplanes derived from the linear feasibility (and attractivity) constraints. Consequently, there need not always be a single candidate 
optimal constraint set sequence within each region of the partition. Indeed, if the remaining number of candidate optima in $\mathcal{O}_{l}$ is unacceptably large for some region $\mathcal{Z}_{l} \in \mathcal{P}_{Z}$, one still has the option to proceed by partitioning the polyhedral region $\mathcal{Z}_{l}$ further, either utilizing the (possibly nonlinear) surfaces derived from the optimality conditions or some approximating hyperplanes. In the optimal constrained LQR (Bemporad et al. 1999) these optimality conditions are characterized by the Lagrange multipliers, which are affine functions of $(x, g, h)$ which lead to hyperplanes. Hence, in the optimal constrained LQR, where there are no restrictions on the allowed switching between active constraint set sequences on the horizon, the exact partition is a union of polyhedra. This is in contrast to the suboptimal approach where there may also be quadratic surfaces in the underlying exact partitioning of the PWL feedback. Whether one wants to pursuit a more detailed partition also utilizing quadratic surfaces will depend on several factors. For the purpose of stability and performance analysis (see section 5 ) is it unclear how a partition with possibly non-convex quadratic surfaces might be utilized. On the other hand, for efficient real-time implementation of the PWL controller introducing such quadratic surfaces may be appealing. However, there is a fairly complex tradeoff involing both real-time computer memory versus processing capacity and the computations required for determining the region associated with the current state and constraint limits versus the computations required for comparing a small number of affine feedbacks with respect to optimality in real-time. A detailed investigation of this topic is outside the scope of the present work, but we note that the framework developed in this work will support a wide range of alternative approaches for real-time implementation.

\section{Stability and Performance Analysis}

In this section we analyse the stability and performance properties of the PWL suboptimal constrained LQR. Due to the suboptimality these properties are not known a priori, and should be computed for each particular control design. We consider the possibly uncertain underlying continuous-time system (1), where $A_{c}$ and $B_{c}$ are matrices

$$
\begin{aligned}
& A_{c} \in \operatorname{Co}\left(A_{1}^{c}, A_{2}^{c}, \ldots, A_{N_{c}}^{c}\right) \\
& B_{c} \in \operatorname{Co}\left(B_{1}^{c}, B_{2}^{c}, \ldots, B_{N_{c}}^{c}\right)
\end{aligned}
$$

i.e. the system is described by the linear differential inclusion

$$
\dot{x} \in \operatorname{Co}\left\{A_{i}^{c} x+B_{i}^{c} u, \mid i \in\left\{1,2, \ldots, N_{c}\right\}\right\}
$$

In this section we assume for simplicity that the constraint limits $(g, h)$ are fixed.

\subsection{Closed loop dynamics}

Due to the characterization of the actitity sets in section 4 , we now have the following set of candidate closed loop dynamics within each projected polyhedral region $\mathcal{X}_{l}(g, h)$ in some partition $\mathcal{P}_{Z}$ of $Z$ :

$$
\dot{x} \in\left\{A_{i}^{c} x+B_{i}^{c} E_{1} \tilde{u}_{k}^{*}(x, g, h) \mid k \in \mathcal{O}_{l}, i \in\left\{1,2, \ldots, N_{c}\right\}\right\}
$$

where it is established in section 2.3 that $\tilde{u}_{k}^{*}(x, g, h)$ is an affine function of $x$. Eq. (97) is a PWL differential inclusion. In the subset of the state space $X \subset R^{n}$ where a feasible or relaxed feasible feedback exists, we have the PWL differential inclusion

$$
\dot{x} \in\left\{A_{i}^{c} x+B_{i}^{c} E_{1} \tilde{u}_{k}^{*}(x, g, h) \mid k \in \mathcal{O}_{l(x, g, h)}, i \in\left\{1,2, \ldots, N_{c}\right\}\right\}
$$

where $l(x, g, h)$ defines the unique region of the state space partition such that $x \in \mathcal{X}_{l(x, g, h)}(g, h)$. The PWL differential inclusion (98) contains the PWL differential equation for the actual closed loop dynamics

$$
\dot{x}=A_{c} x+B_{c} E_{1} \tilde{u}_{k^{*}(x, g, h)}^{*}(x, g, h)
$$

The reasons why we characterize the closed loop dynamics by an inclusion (98) rather than the equation (99) are: 
- The framework allows time-varying uncertainty in the system matrices $A_{c}$ and $B_{c}$ to be incorporated in the analysis.

- The polyhedral state space partition it not always sufficiently rich or fine to ensure that a single affine state feedback is optimal within each region. This is due to computational complexity, but also due to the suboptimality of the design approach.

In this section we derive stability conditions and performance bounds that gives (possibly conservative) guarantees on the closed loop system's stability and performance. Exploiting the PWL nature of the closed loop system, we utilize recently developed computational tools for PWL system analysis based on LMIs (linear matrix inequalities) (Johansson and Rantzer 1998, Rantzer and Johansson 1997, Hedlund and Johansson 1999) allowing us to compute quadratic and piecewise quadratic (PWQ) Lyapunov functions and upper/lower bounds on the cost function of the controller. PWQ functions is a particularly interesting class of functions since at least for the optimal LQR with constraints, it is clear that the optimal cost function $V(x)$ is indeed PWQ since the optimal feedback is PWL (Bemporad et al. 1999).

To fit the framework of (Johansson and Rantzer 1998), we make some changes in notation and rewrite (97) in the form

$$
\dot{x} \in\left\{A_{k, i} x+a_{k, i} \mid k \in \mathcal{O}_{l}, i \in\left\{1,2, \ldots, N_{c}\right\}\right\}, \quad x \in \mathcal{X}_{l}
$$

where we have defined $A_{k, i}=A_{i}^{c}+B_{i}^{c} E_{1} K_{k, 2}, a_{k, i}=B_{i}^{c} E_{1}\left(K_{k, 1}^{g} g+K_{k, 1}^{h} h\right)$, and written $\mathcal{X}_{l}$ instead of $\mathcal{X}_{l}(g, h)$ for simplicity. We also define $\bar{x}=\left(x^{T}, 1\right)^{T}$ and

$$
\bar{A}_{k, i}=\left(\begin{array}{cc}
A_{k, i} & a_{k, i} \\
0_{1 \times n} & 0
\end{array}\right)
$$

which leads to the equivalent closed loop dynamics

$$
\dot{\bar{x}} \in\left\{\bar{A}_{k, i} \bar{x} \mid k \in \mathcal{O}_{l}, i \in\left\{1,2, \ldots, N_{c}\right\}\right\}, \quad x \in \mathcal{X}_{l}
$$

\subsection{Lyapunov functions}

Define the index sets $\mathcal{I}=\left\{1,2, \ldots, N_{P}\right\}, \mathcal{I}_{0}=\left\{l \in \mathcal{I} \mid 0 \in \mathcal{X}_{l}\right\}$ and $\mathcal{I}_{1}=\mathcal{I}-\mathcal{I}_{0}$. Thus, $\mathcal{I}_{0}$ contains the indices to the regions that contain the origin. In general, we will assume $g, h>0$ such that the origin is an admissible state and there exists a single region (corresponding to the unconstrained classical LQR) that contains the origin as an interior point. $\mathcal{I}_{1}$ contains indices to the remaining regions.

Lemma 7 Suppose $g, h>0$. Then $\mathcal{I}_{0} \neq \emptyset$, and for all $l \in \mathcal{I}_{0}, \mathcal{X}_{l}$ contains an open neighbourhood of the origin.

Since the regions $\mathcal{X}_{l}$ are polyhedral, one can construct matrices $\bar{E}_{l}=\left(E_{l}, e_{l}\right)$ and $\bar{F}_{l}=\left(F_{l}, f_{l}\right)$ with $e_{l}=f_{l}=0$ for $l \in \mathcal{I}_{0}$ such that

$$
\begin{array}{ll}
\bar{E}_{l} \bar{x} \geq 0, & x \in \mathcal{X}_{l}, l \in \mathcal{I} \\
\bar{F}_{l} \bar{x}=\bar{F}_{j} \bar{x}, & x \in \mathcal{X}_{l} \cap \mathcal{X}_{j}, j, l \in \mathcal{I}
\end{array}
$$

Hence, the parameterized function $V^{\prime}: R^{n} \rightarrow R$ defined by

$$
V^{\prime}(x)=\bar{x}^{T} \bar{P}_{l} \bar{x}, \quad \bar{P}_{l}=\bar{F}_{l}^{T} T \bar{F}_{l}
$$

is piecewise quadratic and continuous, see (Johansson 1999) for proofs and for details on how to construct the continuity matrices $\bar{F}_{l}$ and cell boundings $\bar{E}_{l}$ based on the partition $\mathcal{X}_{l}, l \in \mathcal{I}$ such that $V^{\prime}$ is continuous. Note that for $l \in \mathcal{I}_{0}, e_{l}=f_{l}=0$ and it follows that $V^{\prime}(0)=0$ and the last row of the matrices and vectors are trivial and can be removed.

Now, the following result follows immediately from Lemma 7 and (Johansson and Rantzer 1998): 
Theorem 4 (Exponential stability) Suppose $g, h>0$ and consider symmetric matrices $T, U_{l, k, i}$ and $W_{l, k, i}$ such that $U_{l, k, i}$ and $W_{l, k, i}$ have nonnegative entries. Suppose

$$
\begin{aligned}
A_{k, i}^{T} P_{l}+P_{l} A_{k, i}+E_{l}^{T} U_{l, k, i} E_{l} & <0, & l \in \mathcal{I}_{0}, k \in \mathcal{O}_{l}, i \in\left\{1,2, \ldots, N_{c}\right\} \\
P_{l}-E_{l}^{T} W_{l, k, i} E_{l} & >0, & l \in \mathcal{I}_{0}, k \in \mathcal{O}_{l}, i \in\left\{1,2, \ldots, N_{c}\right\} \\
\bar{A}_{k, i}^{T} \bar{P}_{l}+\bar{P}_{l} \bar{A}_{k, i}+\bar{E}_{l}^{T} U_{l, k, i} \bar{E}_{l} & <0, & l \in \mathcal{I}_{1}, k \in \mathcal{O}_{l}, i \in\left\{1,2, \ldots, N_{c}\right\} \\
\bar{P}_{l}-\bar{E}_{l}^{T} W_{l, k, i} \bar{E}_{l} & >0, & l \in \mathcal{I}_{1}, k \in \mathcal{O}_{l}, i \in\left\{1,2, \ldots, N_{c}\right\}
\end{aligned}
$$

Then all trajectories $x(t)$, with initial conditions $x(0)$ in the largest invariant set contained in $X$, satisfying the inclusion (100) tends to zero exponentially.

Note that (106)-(109) defines a set of linear matrix inequalities (LMIs) in the matrices $T, U_{l, k, i}$ and $W_{l, k, i}$. The matrix $T$ parameterizes the piecewise quadratic Lyapunov function candidate (105), while the matrices $U_{l, k, i}$ and $W_{l, k, i}$ arise due to application of the $\mathcal{S}$-procedure (Boyd et al. 1994) in order to reduce conservativeness by exploiting the fact that the constitutent local affine dynamics are known to be active in polyhedral regions. Thus, any matrix $T$ that is feasible with respect to (106)-(109) defines a piecewise quadratic Lyapunov function.

\section{Double integrator example, Lyapunov function}

A PWQ Lyapunov function (computed as a feasible solution to (106)- (109)) for the constrained LQR for the double integrator with boundary layers is illustrated in Figure 12.

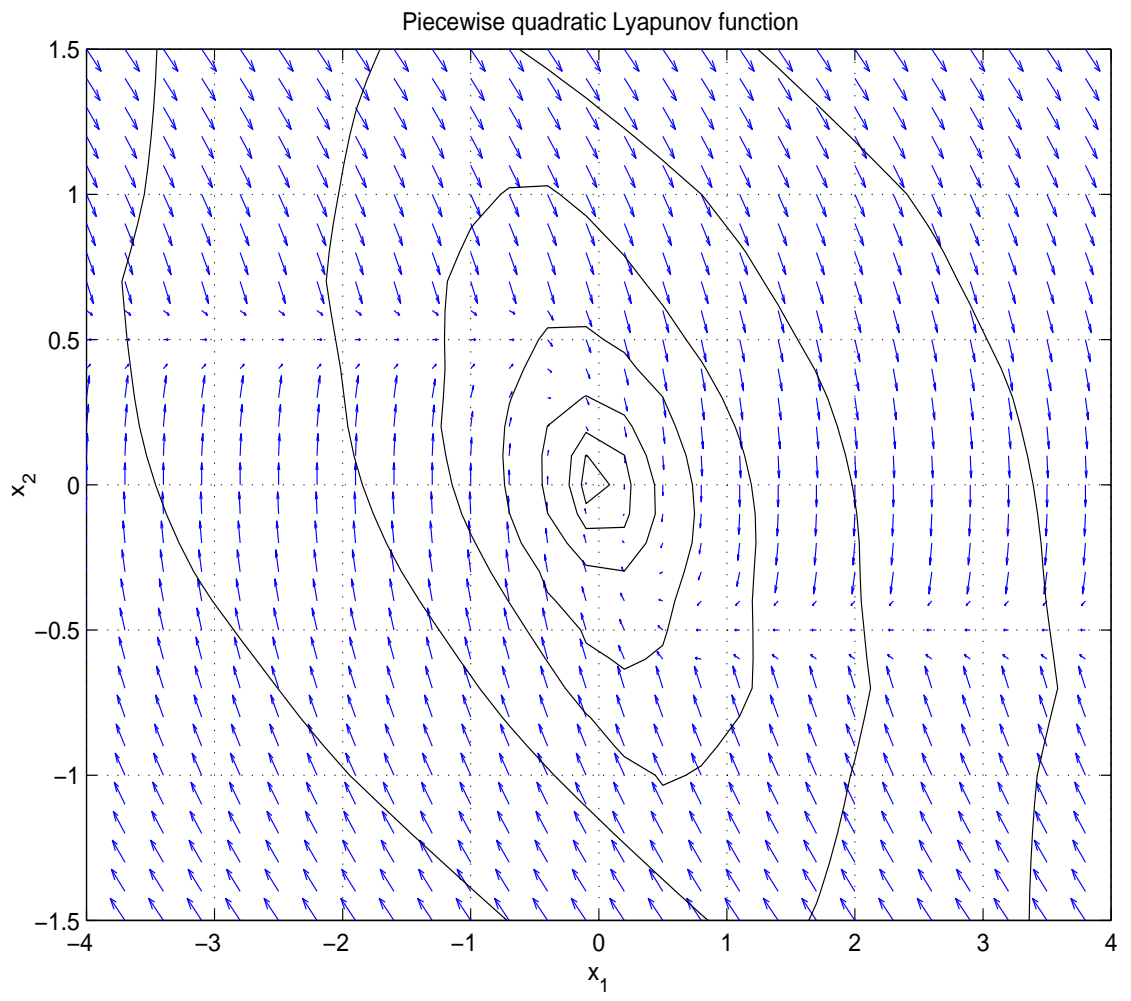

Figure 12: PWQ Lyapunov function for the constrained LQR for the double integrator with boundary layers. 


\subsection{Upper and lower bounds on cost function}

Define the closed loop performance of the suboptimal constrained LQR as follows:

$$
\hat{J}_{c}(x(0))=\int_{0}^{\infty}\left(x^{T}(t) Q x(t)+\left(u^{*}(t)\right)^{T} R u^{*}(t)\right) d t
$$

Next, define for $k \in \mathcal{O}_{l}, l \in \mathcal{I}_{0}$

$$
Q_{k}=Q+K_{k, 2}^{T} R K_{k, 2}
$$

and define for $k \in \mathcal{O}_{l}, l \in \mathcal{I}_{1}$

$$
\bar{Q}_{k}=\left(\begin{array}{cc}
Q+K_{k, 2}^{T} R K_{k, 2} & K_{k, 2}^{T} R\left(K_{k, 1}^{g} g+K_{k, 2}^{h} h\right) \\
\left(K_{k, 1}^{g} g+K_{k, 1}^{h} h\right)^{T} R K_{k, 2} & \left(K_{k, 1}^{g} g+K_{k, 2}^{h} h\right)^{T} R\left(K_{k, 1}^{g} g+K_{k, 1}^{h} h\right)
\end{array}\right)
$$

An upper bound on the performance is now given by the following theorem:

Theorem 5 (Upper bound on performance) Suppose $g, h>0$, and let $x(t) \in X$ with $x(\infty)=0$ be a trajectory of the closed loop system (99). If there exists symmetric matrices $T$ and $U_{l, k, i}$ such that $U_{l, k, i}$ have nonnegative entries and

$$
\begin{aligned}
A_{k, i}^{T} P_{l}+P_{l} A_{k, i}+Q_{k}+E_{l}^{T} U_{l, k, i} E_{l} & <0, \quad l \in \mathcal{I}_{0}, k \in \mathcal{O}_{l}, i \in\left\{1,2, \ldots, N_{c}\right\} \\
\bar{A}_{k, i}^{T} \bar{P}_{l}+\bar{P}_{l} \bar{A}_{k, i}+\bar{Q}_{k}+\bar{E}_{l}^{T} U_{l, k, i} \bar{E}_{l} & <0, \quad l \in \mathcal{I}_{1}, k \in \mathcal{O}_{l}, i \in\left\{1,2, \ldots, N_{c}\right\}
\end{aligned}
$$

then

$$
V(x(0)) \leq \hat{J}_{c}(x(0)) \leq \bar{x}^{T}(0) \bar{P}_{l(x(0), g, h)} \bar{x}(0)
$$

Proof. Note that

$$
\hat{J}_{c}(x(0)) \leq \int_{0}^{\infty} \max _{k \in \mathcal{O}_{l(x(t), g, h)}}\left(\bar{x}^{T}(t) \bar{Q}_{l(x(t), g, h)} \bar{x}(t)\right) d t
$$

and the result follows from (Rantzer and Johansson 1997, Johansson 1999) and Lemma 7.

Consequently, any continuous piecewise quadratic function (105) where $T$ satisfies the LMIs (113)(114) is an upper bound on the performance achieved by the suboptimal constrained LQ controller. It therefore makes sense to minimize $T$ (in some sense) subject to feasibility of the LMIs (113)-(114). Mathematically, this criterion is formulated as

$$
\min _{T, U_{l, k, i}}\left(\sum_{l \in \mathcal{I}_{0}} \xi_{l}^{T} P_{l} \xi_{l}+\sum_{l \in \mathcal{I}_{1}} \bar{\xi}_{l}^{T} \bar{P}_{l} \bar{\xi}_{l}\right)
$$

subject to (113)-(114). The point $\xi_{l}$ is assumed to be an interior point in the region $\mathcal{X}_{l}$. Note that the objective function in (117) is linear w.r.t. the elements of $T$ and this is therefore a convex problem. A lower bound on the achieved performance can be computed in an analogous manner by maximizing $T$ (similar to (117)) subject to reversed LMIs, see (Rantzer and Johansson 1997).

Double integrator example, upper and lower bound on cost

Upper and lower bounds on the cost are illustrated in Figure 13.

\section{Conclusions}

A suboptimal strategy for explicit offline design of LQ controllers subject to state and input constraints is derived. The resulting PWL controller structure is explored and exploited for stability and performance analysis, as well as efficient real-time implementation. 


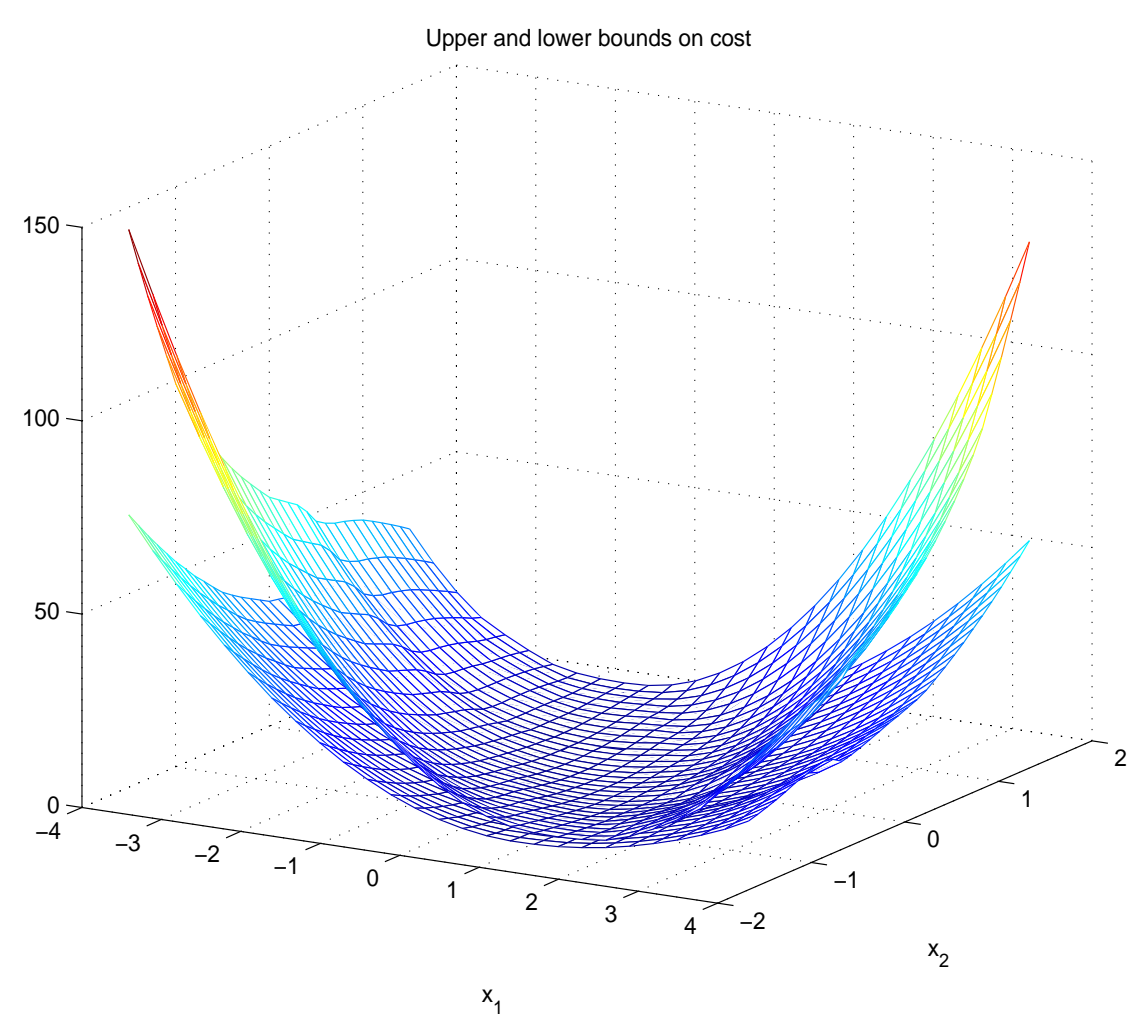

Figure 13: Upper and lower bounds on the cost for the constrained LQR for the double integrator with boundary layers.

The present approach provides a practical and rigorous framework for design, analysis and efficient real-time implementation of controllers that are explicitly designed to satisfy constraints on the states and inputs. One of the main reasons why model predictive control (which in many formulations can be viewed as a non-explicit suboptimal LQR strategy) is popular in industry, is its ability to handle constraints in an efficient manner. So far its success has mainly been limited to process control (Qin and Badgwell 1996), due to the requirements in terms of computer processing capacity and software complexity imposed by the real-time optimization. The present results allow implementation of a similar constrained control strategy at drastically higher sampling rates and reduced software complexity, at least for problems where the real-time computer memory or offline processing capacity does not impose limitations. Preliminary real-time implementations and experiments with standard real-time hardware suggests that typical processing times are in the $\mu s$ to $m s$ range for small and medium-sized problems with a few inputs, states and constraints. Hence, the present approach is suitable for a wide range of application areas. An important current research direction is therefore to streamline the real-time computational implementation, in addition to reducing the computational complexity of the offline analysis and partitioning algorithms.

\section{Acknowlegements}

This work of the first and second authors was in part supported by the European Commission under the ESPRIT Long Term Research project $28104 \mathrm{H}^{2} \mathrm{C}$.

\section{References}

Bellman, R. (1957). Dynamic Programming. Princeton University Press, New Jersey. 
Bemporad, A., M. Morari, V. Dua and E. N. Pistikopoulos (1999). The explicit linear quadratic regulator for constrained systems. Technical Report AUT99-16. Automatic Control Laboratory, ETH Zurich, Switzerland. http://www.control.ethz.ch/pub/publications/AUTAr-99-33.pdf.

Boyd, S., L. El Ghaoui, E. Feron and V. Balahrishnan (1994). Linear Matrix Inequalities in System and Control Theory. SIAM, Philadelphia.

Branicky, M. S. (1998). Multiple Lyapunov functions and other analysis tools for switched and hybrid systems. IEEE Trans. Automatic Control 43, 475-482.

Chisci, L. (1999). Fast algorithm for constrained infinite horizon LQ problem. Int. J. Control 72, 1020-1026.

Chmielewski, D. and V. Manousiouthakis (1996). On constrained infinite-time linear quadratic optimal control. Systems and Control Letters 29, 121-129.

Hedlund, S. and M. Johansson (1999). A toolbox for computational analysis of piecewise linear systems. In: Proceedings of European Control Conference, Karlsruhe.

Johansson, M. (1999). Piecewise Linear Control Systems. PhD thesis. Automatic Control Department, Lund Institute of Technology, Sweden.

Johansson, M. and A. Rantzer (1998). Computation of piecewise quadratic Lyapunov functions for hybrid systems. IEEE Trans. Automatic Control 43, 555-559.

Keerthi, S. S. and E. G. Gilbert (1988). Optimal infinite horizon feedback laws for a general class of constrained discrete-time systems: Stability and moving horizon approximations. $J$. Optimization Theory and Applications 57, 265-293.

Korf, R. E. (1990). Real-time heuristic search. Artificial Intelligence pp. 189-211.

Morse, A. S., Ed.) (1997). Control Using Logic Based Switching. Springer-Verlag, London.

Qin, S. J. and T. A. Badgwell (1996). An overview of industrial model predictive control technology. Preprint CPC-V, Lake Tahoe.

Rantzer, A. and M. Johansson (1997). Piecewise linear quadratic optimal control. In: Proc. American Control Conference. http://www.control.lth.se/ rantzer/publications/pwLQR.pdf.

Rawlings, J. B. and K. R. Muske (1993). The stability of constrained receding horizon control. IEEE Trans. Automatic Control 38, 1512-1516.

Scokaert, P. O. M. and J. B. Rawlings (1998). Constrained linear quadratic regulation. IEEE Trans. Automatic Control 43, 1163-1169.

Slotine, J.-J. E. (1984). Sliding controller design for non-linear systems. Int. J. Control 40, 421-434.

Sznaier, M. and M. J. Damborg (1987). Suboptimal control of linear systems with state and control inequality constraints. In: Proc. 26th Conf. Decision and Control. pp. 761-762.

Sznaier, M. and M. J. Damborg (1990). Heuristically enhanced feedback control of constrained discrete-time linear systems. Automatica 26, 521-532.

Wredenhagen, G. F. and P. R. Belanger (1994). Piecewise-linear LQ control for systems with input constraints. Automatica 30, 403-416. 